\title{
Research Square \\ Fast and Effective Catalytic Degradation of an Organic Dye by Eco-friendly Capped ZnS and Mn doped ZnS nanocrystals
}

\section{Sabri Ouni}

Universite de Kairouan

Naim Bel Haj Mohamed ( $\square$ naimhajmed@gmail.com )

Universite de Kairouan https://orcid.org/0000-0003-2952-4265

Noureddine Chaaben

University of Monastir: Universite de Monastir

\section{Adrian Bonilla-Petriciolet}

Aguascalientes Institute of Technology: Instituto Tecnologico de Aguascalientes

Mohamed Haouari

University of Monastir: Universite de Monastir

\section{Research Article}

Keywords: ZnS-TGA, eco-friendly nanocrystals, Azo dye, photodegradation, Wastewaters

Posted Date: November 15th, 2021

DOI: https://doi.org/10.21203/rs.3.rs-766624/v1

License: (c) (i) This work is licensed under a Creative Commons Attribution 4.0 International License. Read Full License

Version of Record: A version of this preprint was published at Environmental Science and Pollution Research on January 14th, 2022. See the published version at https://doi.org/10.1007/s11356-02117860-1. 
3

Fast and effective catalytic degradation of an organic dye by eco-friendly capped $\mathrm{ZnS}$ and Mn doped $\mathrm{ZnS}$ nanocrystals

Sabri Ouni ${ }^{\mathrm{a}}$,Naim Bel Haj Mohamed ${ }^{\mathrm{b}}$, Noureddine Chaaben ${ }^{\mathrm{c}}$, Adrián Bonilla-Petriciolet ${ }^{\mathrm{d}}$, Mohamed Haouari ${ }^{\mathrm{a}}$

${ }^{a}$ Laboratory of Advanced Materials and Interfaces (LIMA),Faculty of Sciences of Monastir, University of Monastir, Avenue of the environment, 5019 Monastir, Tunisia

${ }^{b}$ Laboratory of Spectroscopic Characterization and Optical Materials (LaSCOM), University of Sfax, Faculty of Sciences B.P. 1171, 3000, Sfax-Tunisia

${ }^{c}$ Research unit on hetero-Epitaxies and Applications ; Faculty of Sciences of Monastir, University of Monastir, Avenue of the environment, 5019 Monastir, Tunisia IInstituto Tecnológicode Aguascalientes, Aguascalientes, 20256, México

*Corresponding authors: E-mail:naimhajmed@gmail.com; Sabriouni1995@gmail.com 


\section{Abstract:}

Undoped and Mn-doped $\mathrm{ZnS}$ nanocrystals encapsulated with thioglycolic acid were synthetized and characterized with different techniques, and finally tested in the photodegradation of a methyl orange in aqueous solution under UV and sunlight irradiations. FTIR and X-ray diffraction results confirmed the functionalization of these nanocrystals surface by thioglycolic acid and the formation of crystalline structures of $\mathrm{ZnS}$ and $\mathrm{Mn}$-doped $\mathrm{ZnS}$ with cubic and hexagonal phases. Calculated average size of $\mathrm{ZnS}$ nanocrystals was in the range of $2-3 \mathrm{~nm}$. It was observed a blue shift of the absorbance threshold and the estimated bandgap energies were higher than that of Bulk $\mathrm{ZnS}$ thus confirming the quantum confinement effect of charge carriers. Photoluminescence spectra of $\mathrm{ZnS}$ nanocrystals exhibited emission in the range of 410- $490 \mathrm{~nm}$ and the appearance of an additional emission band around $580 \mathrm{~nm}(2.13 \mathrm{eV})$ connected to the ${ }^{4} T_{1} \rightarrow{ }^{6} A_{1}$ transition of the $\mathrm{Mn}^{2+}$ ions. Photodegradation of methylene orange with undoped and Mn-doped ZnS-TGA nanocrystals was investigated. Dye adsorption prior to photocatalysis using nanocrystals was studied via kinetic experiments and statistical physics models. The maximum dye adsorption capacity on doped ZnS-TGA was $~ 26.98 \mathrm{mg} / \mathrm{g}$. The adsorption kinetic was found to follow the pseudosecond-order kinetic model.According to the statistical physics results, the calculated adsorption energy was $22.47-23.47 \mathrm{~kJ} / \mathrm{mol}$ and it showed that the dye adsorption was associated to the hydrogen interaction where the removal process was feasible and multimolecular. The photocatalytic activity of undoped $\mathrm{ZnS}$ nanoparticles under UV irradiation showed better efficiency than doped nanocrystals thus indicating that manganese doping generated a dropping of the photocatalytic degradation of the dye. Dye degradation efficiency of $81.37 \%$ using ZnS-TGA nanocrystals was achieved after $6 \mathrm{~min}$, which indicated that ZnMnS-TGA nanocrystals may be considered as an alternative low cost and environmental friendly material for facing water pollution caused by organic compounds via photodegradation processes.

Keywords: ZnS-TGA; eco-friendly nanocrystals; Azo dye; photodegradation, Wastewaters

\section{Introduction:}

Semiconductors nanoparticles (NPs) have found numerous applications in different technological fields going from photovoltaic devices to medical imaging, lasers, LEDs and photocatalysis (Dao 2020; Rtimi et al. 2021; Meikle et al. 2020; Long et al. 2020; Su et al. 2020; Li et al. 2019; Daskalakis et al. 2020; Rtimi et al. 2021). These applications are supported by their unique properties as compared to their bulk counterpart, which arise from 
the confinement of the charge carriers as a result of their small sizes. This quantum confinement effect enlarges the energy bandgap, which makes the electronic and optical properties size dependent allowing the control of the absorption and the emission wavelengths of these materials. Additionally, semiconductor NPs have a large surface to volume ratio, which is responsible for a significant change in their physical and chemical properties. Indeed, the surface atoms are less coordinated than internal ones. This makes these NPs lesser stable against aggregation and more reactive with their environment in comparison to bulk materials. Additionally, the dangling bonds and surface defects introduce energy levels in the bandgap, which are usually responsible for non-radiative transitions and the emission quenching of semiconductor NPs. To prevent the agglomeration and the drawbacks of surface defects, NPs must be grown within a protecting medium such as glass and polymer or enrobed by encapsulating molecules that should be well fixed at their surface.

Recently, semiconductor NPs have emerged as promising photocatalysts to produce hydrogen by water dissociation or to degrade organic pollutants in wastewater being more effective than the conventional methods (Buthiyappan et al. 2016). However, these NPs must be dispersible in water to maximize their performance. Accordingly, the colloidal wet chemical synthesis has emerged as the most popular safe way to produce, at large scale and cost-effective, watersoluble semiconductor NPs with narrow size dispersion and promising optical and electronic properties (Jaldurgam et al. 2021).

Among the different semiconducting nanostructures, ZnS NPs are recognized as environmentally friendly and effective photocatalyst for the degradation of organic pollutants in wastewaters. This is due to their nontoxicity when they are used in small amounts and their unique electronic and optical properties. Moreover, ZnS NPs have a high absorption coefficient required for solar light harvesting, which is the first step for the photodegradation of organic pollutants at industrial level. They have also a high negative reduction-oxidation potential of excited electrons due to their higher conduction band position in an aqueous solution as compared to other extensively studied photocatalysts (Yang et al. 2014). Additionally, these NPs are able to rapidly generate great density of electron-hole pairs under photoexcitation because of their direct bandgap (Arao et al. 2009), and they show competitive photocatalytic activity due to trapped holes arising from surface defects (Saenger et al. 1998). The photocatalytic activity of these semiconductor NPs is due to the formation of $\mathrm{OH}^{*}$ and $\mathrm{O}_{2}^{-}$ free radicals that results from the reaction of the photo-generated holes and electrons with water and free oxygen molecules, respectively. However, $\mathrm{ZnS}$ nanocrystals (NCs) that belong 
to large bandgap semiconductors $(3.7 \mathrm{eV})$ can absorb only UV radiation, which reduces their photoactivity. One way to enhance the efficiency of these nanostructures and to improve their visible light harvesting efficiency is to extend the wavelength range photoactivity towards visible light region of solar spectrum by doping them by transition metal ions such as $\mathrm{Mn}^{2+}$, $\mathrm{Ni}^{2+}, \mathrm{Cu}^{2+}$ (Kostrov et al. 2020; Kabachii et al. 2021; Wang et al. 2017; Chauhan et al. 2014; Othman et al. 2020). The doping impurities introduce trapping levels in the bandgap that reduces the carrier's recombination probability and allows them to diffuse faster to the NCs surface. With the aim to obtain an efficient, low- cost and eco-friendly nanocatalysts that can be used at industrial scale, this paper reports the synthesis and application of $\mathrm{ZnS}$ and Mndoped ZnS NPs via colloid method. The physico-chemical properties of these nanostructures were investigated and their photocatalytic activity to decompose methylene orange (MO) dye was evaluated by conducting degradation experiments under UV and sunlight.

\section{Methodology}

\subsection{Chemicals}

All chemicals were purchased from Sigma-Aldrich. They were of analytical grade and used without any purification process. They included zinc (II) acetate dihydrate (Sigma-Aldrich, $\mathrm{Zn}\left[\mathrm{CH}_{3} \mathrm{COO}\right]_{2} \cdot 2 \mathrm{H}_{2} \mathrm{O} \geq 98 \%$ ), manganese sulfate $\left(\mathrm{MnSO}_{4}\right)$, sodium sulfide (Sigma-Aldrich, $\mathrm{Na}_{2} \mathrm{~S} \geq 98 \%$ ), thioglycolic acid (TGA) (Sigma-Aldrich, $\mathrm{HSCH}_{2} \mathrm{COOH}$, 97\%), sodium hydroxide $(\mathrm{NaOH}, 99 \%)$ and methylene orange dye (Sigma-Aldrich, $\mathrm{C}_{14} \mathrm{H}_{14} \mathrm{~N}_{3} \mathrm{NAO}_{3} \mathrm{~S} \geq 50 \%$, $\mathrm{MW}=327.33 \mathrm{~g} / \mathrm{mol}$ ). All aqueous solutions were prepared with ultra-pure water.

\subsection{Synthesis of TGA capped $\mathrm{ZnS}$ nanoparticles}

Undoped $\mathrm{ZnS}$ and Mn-doped $\mathrm{ZnS}$ NCs were prepared using the colloidal hydrothermal reaction method(Ouni et al. 2021), see Figure 1. The experimental synthesis protocol is briefly described as follows. An aqueous solution was prepared by mixing dihydrated zinc acetate $(2 \mathrm{mmol})$ with TGA $(5 \mathrm{mmol})$ in $100 \mathrm{~mL}$ of water. Manganese sulphate was added at different percentages in weight $(4,8$ and $12 \%)$ with respect to zinc for each synthesis. The $\mathrm{pH}$ of the resulting mixture was adjusted to 11 by drop addition of $\mathrm{NaOH}(1 \mathrm{M})$ with continuous agitation under $\mathrm{N}_{2}$ for $30 \mathrm{~min}$. The second step of this synthesis protocol was based on the injection of $\mathrm{S}^{2-}(0.08 \mathrm{mmol})$ at room temperature into the solution containing $\mathrm{Zn}^{2+}$-TGA complexes. The mixture was then heated at $100^{\circ} \mathrm{C}$ for $3 \mathrm{~h}$. Finally, the reaction mixture was cooled to room temperature to stop the NCs growth. After solution cooling and drying, 
undoped and Mn-doped ZnS NCs were purified by precipitation with ethanol, washed two times with ethanol and stored in vacuum at room temperature.

\subsection{NCs characterization}

X-ray diffraction (XRD) characterization was performed using Philips X'Pert PRO MPD diffractometer equipped with a $\mathrm{Cu} \mathrm{K} \alpha$ source of wavelength $\lambda=1,542 \AA$. Transmission electron microscopy (TEM) images were obtained using a FEI Tecnai G2 electron microscope operated at an accelerating voltage of $200 \mathrm{kV}$ and equipped with Energy Dispersive X-ray (EDX) for element chemical analysis. Samples were analyzed by placing a drop of aqueous suspension of particles onto a carbon film on a copper grid. Fourier transform infrared (FTIR) spectra were recorded at room temperature with a Perkin Elmer version 5.3 within the wavenumber range of $400-4000 \mathrm{~cm}^{-1}$ and using $\mathrm{KBr}$ pellets. UV-visible absorption spectra were obtained at room temperature in the range of 200-800 $\mathrm{nm}$ using a SPECORD 210 Plus spectrophotometer with quartz cuvettes. Photoluminescence (PL) spectra were recorded at room temperature using conventional photoluminescence setup and a helium cadmium laser as excitation source $\left(\lambda_{\text {exc }}=325 \mathrm{~nm}\right)$.

\subsection{Dye adsorption experiments: kinetics and isotherms}

Dye adsorption experiments were performed at $300 \mathrm{~K}$ in a batch system. In a typical experiment, $50 \mathrm{~mL}$ of dye solution with different initial concentrations and $50 \mathrm{mg}$ of nanocatalyst (m) were used for these studies. The adsorption experiments were carried out at $\mathrm{pH} 7$. Adsorption parameters like time contact and dye concentration were investigated. Kinetic studies were performed with MO dye concentrations from 10 to $50 \mathrm{mg} / \mathrm{L}$ with a sampling period of 120 min and magnetic stirring in the dark to measure the MO adsorption capacities. The concentration of dye solution was determined using SPECORD 210 Plus UV-Vis spectrometer at $465 \mathrm{~nm} . \mathrm{MO}$ dye adsorption capacities Qe $(\mathrm{mg} / \mathrm{g})$ were calculated using the next equation

$$
Q_{e}=\frac{V\left(C_{0}-C_{e}\right)}{m}
$$

where $\mathrm{C}_{0}$ is the initial dye concentration $(\mathrm{mg} / \mathrm{L}), \mathrm{Ce}$ is the equilibrium dye concentration in the solution $(\mathrm{mg} / \mathrm{L}), \mathrm{V}$ is the dye solution volume $(\mathrm{mL})$ and $\mathrm{W}$ is the weight of the nanoadsorbent (mg). 


\subsection{Photocatalytic degradation tests}

The photocatalytic efficiency of the undoped and Mn-doped ZnS NCs was evaluated via the photodegradation of MO dye as a model molecule of an organic pollutant. Before starting the photocatalytic degradation experiments, the aqueous solutions containing both dye and NCs were submitted to a vigorous magnetic stirring in dark for $2 \mathrm{~h}$ in order to ensure the adsorption/desorption equilibrium (Chan et al. 2011).Then, they were submitted to irradiation by mercury lamp of $160 \mathrm{~W}$ to monitor the MO degradation. Solution samples were taken at regular time intervals and analyzed by UV-visible spectroscopy to determine the absorbance at $465 \mathrm{~nm}$, which corresponded to the maximum absorption of MO molecule. Degradation experiments were also conducted under sunlight.

\section{Results and discussion}

\subsection{Structural and morphological characterization of NCs}

FTIR spectra of the different samples are shown in Figure 2. All these spectra showed an absorption band at $563 \mathrm{~cm}^{-1}$ that was attributed to the stretching vibrations of $\mathrm{Zn}$ S(Amaranatha Reddy et al. 2014). The spectra of ZnS NCs doped with different concentrations of $\mathrm{Mn}^{2+}$ showed the presence of the same absorption bands at wavenumber values close to those recorded with pure $\mathrm{ZnS}$ and no other apparent bands to other vibration modes of doping impurities or other elements were observed. These spectra also showed a wide band at $\sim 3360 \mathrm{~cm}^{-1}$, which was associated to the elongation vibration of $(\mathrm{O}-\mathrm{H})$ groups of TGA ligand(Liu et al. 2008). FTIR spectrum of TGA showed the presence of a characteristic band at $2560 \mathrm{~cm}^{-1}$ related to the $\mathrm{S}-\mathrm{H}$ bond (Dehghan et al. 2018). This absorption band was absent in TGA-capped ZnS NCs spectra thus indicating the rupture of S$\mathrm{H}$ bond and the fixation of the sulfur atom on the NCs surface (Xie et al. 2011). This finding can be explained by the strong interaction of the sulfur electron pair with zinc atoms on the surface (Ouni et al. 2021). In addition, these spectra contained absorption bands around 570$700,850,1429,1508$ and $1627 \mathrm{~cm}^{-1}$ assigned to $(\mathrm{C}-\mathrm{S}),(\mathrm{C}-\mathrm{H}),\left(\mathrm{COO}^{-}\right),(\mathrm{C}=\mathrm{O})$ and $(-\mathrm{OH})$ bonds, respectively (Amirian et al. 2018). These results clearly demonstrated that NCs were well functionalized by TGA ligand, which allowed the control of their growth during synthesis and prevented NCs aggregation (Singh and Chauhan 2009).

Figure 3 shows the XRD pattern of the undoped and Mn-doped ZnS samples. In the case of undoped ZnS NCs, XRD diffraction patterns contained peaks at $2 \theta=28.95,33.76,47.58$ and $55.27^{\circ}$. These peaks were attributed to the diffraction plans (111), (200), (220) and (311) of 
the zinc blende phase (ZB) of $\mathrm{ZnS}$ according to JCPDS (80-0007). However, it was also observed the existence of a diffraction peak at $2 \theta=31.08^{\circ}$ that corresponded to the (101) plan of the wurtzite phase (WZ) of ZnS (JCPDS 80-0020). This structural anomaly made the distinction between the cubic and hexagonal structures very difficult. The most intense peak attributed to (111) suggested that NCs growth in this direction was preferred and confirmed that the structure of ZnS NCs was almost cubic. XRD patterns were adjusted with Gaussian profiles to calculate the full-width-at-half-maximum (FWHM) of identified peaks and these values were used to estimate the crystallite size according to Scherrer formula(Jothibas et al. 2018):

$$
D=\frac{K \lambda}{\beta \cos (\theta)}
$$

Where $D$ is the average crystallite size (nm), $\lambda$ is the wavelength of X-ray (1.5402 $) \mathrm{Cu} \mathrm{K \alpha}$ radiation, $K$ is the shape factor (0.9), $\beta$ is the measured full width at half maximum of the diffraction peak and $\theta$ is the Bragg diffraction angle. The average size of the undoped $\mathrm{ZnS}$ NCs was estimated to be $\sim 7.15 \mathrm{~nm}$, while the lattice parameter was $5.38 \AA$.

For Mn-doped NCs, the peaks at $29.52^{\circ}, 31.82^{\circ}, 47.31^{\circ}$ and $56.12^{\circ}$ were assigned to the plans (002), (101), (110) and (200) of the hexagonal structure according to the JCPDS hexagonal ZnS standard sheet (80-0007). However, the presence of the peak associated with the crystallographic plane (200) was around $34.77^{\circ}$ thus indicating the presence of the cubic phase of ZnS. Similar results were reported for ZnS NCs synthesized by precipitation in an aqueous medium (Chauhan et al. 2014). The estimated crystallite sizes are given in Table 1. NCs size decreased from 7.15 to $2.5 \mathrm{~nm}$ with the increment of Mn doping content up to $12 \%$. This result indicated that $\mathrm{Zn}^{2+}$ ions were replaced by $\mathrm{Mn}^{2+}$ ions in the $\mathrm{ZnS}$ matrix. The reduction of $\mathrm{ZnS}$ NCs size with the doping concentration could be explained by the induced NCs growth and enhancement of surface area to volume ratio in the system (Mote et al. 2013). This result confirmed the incorporation of manganese into the host lattice of $\mathrm{ZnS}$ and the formation of Mn-doped ZnS NCs. However, the Scherrer formula takes into account only the broadening effect of the spectracoming from the different diffracting domains and does not include the intrinsic micro-strain effect created within the lattice by stacking fault, point defect and grain boundary (Poornaprakash et al. 2018; Pandya et al. 2016). Therefore, the change of NCs size may be correlated to different structural parameters including the lattice strain $(\varepsilon)$, dislocation density $(\delta)$ and stacking fault $(S F)$. These parameters can be estimated by using the next formula (Nath et al. 2020; Jothibas et al. 2016; Bel Haj Mohamed et al. 2021) and the calculated values are summarized in Table 1. 


$$
\varepsilon=\frac{\beta \cos (\boldsymbol{\theta})}{4}
$$

$$
\delta=\frac{1}{D^{2}}
$$

$$
S F=\frac{2 \pi^{2}}{45(\tan \theta)^{\frac{1}{2}}} \beta_{h k l}
$$

244

The value of dislocation densitycan be associated to the amount of defects and vacancies in the crystals. Results showed that $\delta$ increased with the doping Mn content from zero to $12 \%$. This was due to the inversely proportional relationship between the dislocation and the size of NCs. Thus, the smallest particles (ZnS: Mn 12\%) have the highest possibility of dislocations since they have a tendency to minimize their higher surface energy (Gaceur et al. 2012). Undoped ZnS NCs with the highest size were the most ordered compared to Mn-doped ZnS. This finding made obvious the effect of doping with Mn on the change of the high orientation plane from (111) to (101) as well as the improvement of crystallinity. Both micro-strain and stacking fault showed the same trends when they decreased with increasing the doping ratio except for the case of Mn 8\%. The decreasing of micro-strain indicated that the Mn-doped $\mathrm{ZnS}$ samples contained lattice defects and vacancies. On the other hand, SF describes the disordering of crystallographic planes. Therefore, the decreasing tendency of SF suggested that the defects increased by substituting zinc by manganese.

Figure 4 shows TEM images of tested samples and the corresponding size distribution histograms. NCs exhibited a spherical shape and were agglomerated probably due to the insufficient ligand amount to cover all ZnS NCs and inhibited the aggregation and formation of large clusters. This agglomeration may be also due to high particles concentrations used in TEM experiments. From the histograms of undoped and Mn (8\%)-doped NCs, it was found that the average NCs diameters were 5.9 and $4.1 \mathrm{~nm}( \pm 0.5 \mathrm{~nm})$, respectively. These results demonstrated that Mn-doping decreased the $\mathrm{ZnS}$ particle size. The inter-planar distance were determined from digital micrograph for undoped and doped $\mathrm{ZnS}$ and it was $0.36 \mathrm{~nm}$, which corresponded to the plane (111) of theZB phase and $0.2 \mathrm{~nm}$ for the plane (101) of the WZ phase, respectively. These results are consistent with the values found by XRD measurements. EDX spectra for ZnS and Mn-doped ZnS NCs are shown in Figure 5. In particular, Figure 5a indicated the presence of $\mathrm{Zn}$ and $\mathrm{S}$ that were the major elemental components with also the presence of Si related to the detector signal. It was observed the presence of the carbon, oxygen and copper peaks that were related to TEM grid and ligand stabilizer. Results of Mn (8\%)-doped NCs (Figure 5b) showed the presence of $\mathrm{Zn}, \mathrm{S}$ and $\mathrm{Mn}$, which confirmed the formation of doped NCs. This diagram also indicated the existence of carbon, oxygen and 
silicon related to TGA ligand and TEM devices. Others peaks related to $\mathrm{Al}, \mathrm{Ca}, \mathrm{K}, \mathrm{Ni}$ and $\mathrm{Fe}$ were also detected probably due to residues coming from the main precursors during the synthesis. Hence, TEM results indicated the formation of undoped and doped ZnS NCs that were properly stabilized by TGA.

\subsection{Optical characterization of NCs}

UV-visible absorption spectra of the different NCs are reported in Figure 6. They showed a blue shift of the absorption threshold when increasing $\mathrm{Mn}^{2+}$ concentration. The absorption threshold was 307, 300, 298 and $290 \mathrm{~nm}$ for 0, 4, 8 and 12\% Mn-doping concentrations, respectively. In accordance with the average sizes obtained from XRD analysis, this behavior was explained by the presence of small Mn-doped $\mathrm{ZnS} \mathrm{NCs,} \mathrm{which} \mathrm{induced} \mathrm{the} \mathrm{widening} \mathrm{of}$ gap energy(Meng et al. 1995).

The Tauc equation was utilized to estimate the gap energy of undoped and Mn-doped $\mathrm{ZnS}$ NCs(Kaur et al. 2015).

$$
\alpha h v=A\left(h v-E_{g}\right)^{n}
$$

where $\alpha$ is the absorption coefficient, $A$ is a constant, $h v$ is the photon energy, $E g$ is the optical gap of NCs and $n$ is a constant with $n=1 / 2$ for direct semiconductors. The optical band gap energy of NCs were estimated from the intercept of the extrapolation of Tauc plots with the X-axis (Figure 7) and the results of the optical gap energies are displayed in Table2.

The calculated value of the optical gap energy of NCs increased from 3.75 to $3.92 \mathrm{eV}$ with increasing manganese doping. This variation in $E_{g}$ was attributed to variations of NCs size by the incorporation of manganese ions in the zinc substitute position within the $\mathrm{ZnS}$ structure. At high doping concentrations, manganese ions caused a significant increment of $E_{g}$. This finding was explained by the Burstein-Moss effect, which demonstrated that the excitation of an electron from the valence band to conduction band in a doped semiconductor required more energy due to the accumulation of electrons in the conduction band caused by the shallow donor states (i.e., the movement of the Fermi level to the conduction band by doping effect)(Brus 1986).

The average NPs sizes were also estimated by using the optical gap energy and the effective mass approximation according to the next equation (Jai Kumar and Mahesh 2017; Mansur and Mansur 2011):

$$
E_{g}^{n}=E_{g}^{b}+\frac{h^{2}}{8 R^{2}}\left(\frac{1}{m_{e}^{*}}+\frac{1}{m_{h}^{*}}\right)-\frac{1.8 e^{2}}{4 \pi \varepsilon R}
$$

where $E_{g}^{n}$ and $E_{g}^{b}$ are the band gap energies of NPs and bulk material, respectively, $m_{e}^{*}$ and $m_{h}^{*}$ are the effective masses of the electron and hole, $e$ is the elementary electric charge and $\varepsilon$ is 
the dielectric constant of $\mathrm{ZnS}$. It was observeda reduction in the size of the doped $\mathrm{ZnS} \mathrm{NCs}$ from 2.3 to $1.9 \mathrm{~nm}$ as the doping rate increased. The difference between the sizes found by absorption spectroscopy and DRX was attributedto the fact that calculation of the latter values were based on the effective mass approximation assuming that the NCs geometry was spherical; while the XRD measurements in polydisperse systems were weighted to the largest particles (Pejjai et al. 2017). Additionally, when the quantum dots are small enough, the carrier's wave functions overlaps with the crystal boundaries thus resulting in energy dependent effective masses (Jai Kumar and Mahesh 2017; Mansur and Mansur 2011). This discrepancy may be also due to the fact that XRD results were obtained for powdered NCs, while absorption measurement involved NCs dispersed in solution.

Figure 8 shows the room-temperature photoluminescence spectra of undoped and Mn-doped ZnS NCs with different Mn concentrations. PL spectra of pure ZnS nanocrystals showed two wide and asymmetrical bands. Accordingly, they were convoluted with three Gaussian curves, see Figure 9. The observed emission spectra may be the result of several emissions transition essentially caused by the size distributions of the $\mathrm{ZnS}$ NCs with a large contribution of surface defects. In fact, the stoichiometric ratio of synthetic precursor $(\mathrm{Zn} / \mathrm{S} \sim 2.5)$ and the synthesis method were responsible for the formation of punctual defects (interstitials, substitutes) or extended (dislocations, aggregates, cavities) within the $\mathrm{ZnS}$ structure. Indeed, the use of excess zinc caused that defects present in the $\mathrm{ZnS}$ NCs correspond to zinc interstitials $\left(\mathrm{Zn}_{\mathrm{i}}\right)$ or sulphur vacancy (Vs) (Peng et al. 2006). For the undoped ZnS NCs, the spectrum was dominated by a band around $426 \mathrm{~nm}$ responsible for the blue luminescence originated from sulfur vacancies Vs (Wang et al. 2011), while the yellow emission at $540 \mathrm{~nm}$ may be due to interstitial zinc atom ( $\left.I_{Z n}\right)$ (Phuong Nguyen et al. 2017). A new orange emission appeared at $580 \mathrm{~nm}$ when inserting $\mathrm{Mn}^{2+}$ ions into the crystal, which was attributed to ${ }^{4} \mathrm{~T}_{1} \rightarrow{ }^{6} \mathrm{~A}_{1}$ transition of $\mathrm{Mn}^{2+}$ ions (Tuan et al. 2018). The band at $\sim 420 \mathrm{~nm}$ persisted and was related to radiative carriers recombination between the sulphur vacancy sites $(\mathrm{Vs})$ and the valence band (Mansur and Mansur 2011). In terms of intensity, the luminescence of the orange emission band increased with the doping level and reached a maximum emission for a concentration of $8 \%$ $\mathrm{Mn}^{2+}$. This result was associated to energy transfer from $\mathrm{ZnS}$ host matrix to $\mathrm{Mn}^{2+}$ ions due to the strong coupling between the $3 \mathrm{~d}^{5}$ electrons of $\mathrm{Mn}^{2+}$ ions and the $\mathrm{ZnS}$ p-electrons (Kolmykov et al. 2014). At high doping concentration, the distance between $\mathrm{Mn}^{2+}$ ions decreased and the dipole-dipole interaction between the electronic spins of two neighboring $\mathrm{Mn}^{2+}$ ions was no longer negligible. Such coupling generated a reduction of the manganese 
emission band with a slight red shift caused by a non-radiative cross-relaxation between these ions (Fang et al. 2010). On the other hand, the decrement of the intensity of the overall emission may be because $\mathrm{Mn}^{2+}$ ions settled at the superficial or interstitial positions of the $\mathrm{ZnS}$ host. Some of these defects acted as non-radiative recombination centers in the host as explained by Mohagheghpour et al.(Mohagheghpour et al. 2009). In order to provide a better understanding of the different emission bands of Mn-doped $\mathrm{ZnS} \mathrm{NCs,} \mathrm{an} \mathrm{energy} \mathrm{level}$ diagram was proposed where the possible radiative and non-radiative transitions of charge carriers are illustrated, see Figure 10.

\subsection{MO dye adsorption and its analysis via advanced statistical physics model}

In recent years, II-VI semiconductors especially zinc sulfur ( $\mathrm{ZnS}$ ) have generated considerable interest in wastewaters treatment due to their nontoxicity, physical and chemical stability and high catalytic efficiency (Chang et al. 2017; Bujňáková et al. 2017; Ajibade et al. 2020). ZnS nano-adsorbents have advantages in terms of their abundance, low-cost, good stability, non-toxicity and good light UV absorption. Therefore, they are considered suitable for adsorption and photocatalysis applications. Herein, a simple colloidal chemical route for the synthesis of undoped and Mn-doped $\mathrm{ZnS}$ nano-adsorbents using thioglycolic acid (TGA) as a stabilizing agent is reported. This synthesis route offers a high purity, low-cost, simple implementation and low energy consumption. The surface functionalization of nanocrystals by TGA molecules modifies their adsorption activities, morphology, particle size, optical properties and mechanical stability (Wieszczycka et al. 2021). It is worth noting that the surface area and small particle size of $\mathrm{ZnS}$ contribute to enhance the adsorption efficiency. Furthermore, the combination of adsorption and photocatalyst is an interesting approach to degrade organic pollutants in wastewaters treatment.

Figure 11 reports the adsorption kinetic of MO dye on undoped and Mn-doped $\mathrm{ZnS}$ nanoadsorbent in dark conditions.Dye adsorption on the nano-adsorbent was fast for the first 5 min, then continued with a slower rate during 10-45 $\mathrm{min}$ and reached the equilibrium condition after approximately $60 \mathrm{~min}$. The experimental kinetic showed that doping with $\mathrm{Mn}$ ions increased the MO adsorption capacity. Mn-doped ZnS NCs showed a better adsorption than undoped ZnS NCs. This can be explained by the decrease in size under the effect of doping, which generated an increase in the surface-to-volume ratio that provided more sites on the surface. The pseudo second order kinetic rate equation (Mohamed et al. 2018; Bouzid et al. 2019; Pang et al. 2020) was used to fit the experimental data (S 1898; Ho and McKay 1999; Pan et al. 2010; Ghasemi et al. 2014): 


$$
Q(t)=\frac{q_{e}^{2} K_{2} t}{1+q_{e} K_{2} t}
$$

373 where $\mathrm{K}_{2}(\mathrm{mg} / \mathrm{gmin})$ is the pseudo second order rate constant of adsorption, $\mathrm{q}_{\mathrm{e}}$ and $\mathrm{Q}(\mathrm{t})$ $374(\mathrm{mg} / \mathrm{g})$ are the adsorption capacity at equilibrium and time t, respectively. Table 3 shows that $375 \mathrm{~K}_{2}$ increased as a function of the Mn doping amount. Overall, the adsorption kinetic of MO on undoped and Mn-doped $\mathrm{ZnS}$ nanocrystals could involve a rate limiting adsorption on the vicinity of Mn-doped $\mathrm{ZnS}$ surface. This process can be associated to an exchange interaction between MO and Mn-doped $\mathrm{ZnS}$ adsorbent surface. This interaction increased with Mn doping in the $\mathrm{ZnS}$.

On the other hand, the experimental isotherm showed that MO adsorption capacity of undoped $\mathrm{ZnS}$ increased from 6.21 to $25.91 \mathrm{mg} / \mathrm{g}$ when the dye concentration also increased from 10 to $50 \mathrm{mg} / \mathrm{L}$, which corresponded to an adsorption efficiency of $64.56 \%$. However, the Mn (12\%)-doped ZnS NCs showed an adsorption efficiency of $71.16 \%$, which proved that the doping with manganese ions enhanced the adsorption. The adsorption capacity of undoped and $\mathrm{Mn}$-doped $\mathrm{ZnS}$ nanocrystals could be due to the ligand on the surface of inorganic TGA particles, which could contribute with positively charged sites on the surface of $\mathrm{ZnS}$ due to the zinc interstitials $\left(\mathrm{Zn}_{\mathrm{i}}\right), \mathrm{Mn}$ defects and sulfur vacancies $(\mathrm{Vs})$ that were generated during the synthesis (such as nonstoichiometric defects and dangling bonds). These sites generated attraction forces with the negatively charged molecule of MO (with sulfonate group R$\mathrm{SO}_{3}{ }^{-}$). The adsorption mechanism of the $\mathrm{MO}$ molecule on $\mathrm{ZnS}$ adsorbent surface, with different doping percentage of Mn, was analyzed with the Hill adsorption model obtained from statistical physics. This model is presented in the Equation (9) (Bouzid et al. 2016, 2019; Mohamed et al. 2018; Pang et al. 2020 ).

$$
Q_{a}=\frac{n_{D} D_{M}}{\left(1+\left(\frac{C_{1 / 2}}{C}\right)^{n_{D}}\right)}
$$

wheren $_{D}$ is the number of MO molecules captured by the adsorption receptor site of adsorbent surface, $\mathrm{D}_{\mathrm{M}}$ is the density of receptor sites and $\mathrm{C}_{1 / 2}$ is the concentration at half-saturation of $\mathrm{MO}$ on the $\mathrm{ZnS}$ adsorbent surface with different doping of Mn. This last parameter can be associated to the adsorption energy using the next expression (Bouzid et al. 2016, 2019; Mohamed et al. 2018; Atrous et al. 2019; Pang et al. 2020; Bel Haj Mohamed et al. 2021; Sghaier et al. 2021). 
402

403

404

405

406

407

408

409

410

411

412

413

414

415

416

417

418

419

420

421

422

423

424

where $\mathrm{C}_{\mathrm{S}}$ is the $\mathrm{MO}$ dye solubility (i.e., $5.20 \mathrm{~g} / \mathrm{L}$ ). MO adsorption data were fitted with this model and their corresponding values are listed in Table 4, while the model fitting is illustrated in Figure 12.

Note that the parameter $n_{D}$ can be used to describe the number of dyes adsorbed molecules per adsorption site and gives also information about the adsorption position. Table 4 showed that $n_{D}=3.73$ indicating that MO dye molecules could be linked or adsorbed via a non-parallel orientation with a multi-molecular adsorption process. MO molecules can interact with the adsorbent surface thus forming an aggregate of three molecules per adsorption site of Mndoped $\mathrm{ZnS}$ nanocrystals. Overall, $\mathrm{n}_{\mathrm{D}}$ decreased with the increment of $\mathrm{Mn}$ amount in the $\mathrm{ZnS}$ host. Dye adsorption capacity at saturation was $25.31 \mathrm{mg} / \mathrm{g}$ and increased with the Mn doping. $\mathrm{Mn}$ in the $\mathrm{ZnS}$ NCs contributed to increase the specific adsorption surface of nanocrystals. The adsorption energy of MO on Mn-doped ZnS nanocrystals surface ranged from 22.47 to $23.47 \mathrm{~kJ} / \mathrm{mol}$ thus corresponding to physical interactions like hydrogen bonding and van der Waals forces, see Table 4.

\subsection{Photocatalytic activity}

Figure 13 shows the absorption spectra of MO solutions using undoped and Mn-doped $\mathrm{ZnS}$ nanocatalysts for various contact times (0-120 min). The maximum absorption peak decreased with increasing irradiation time as a result of the reduction of MO dye concentration in the irradiated solution. It was observed the color disappearance after 6 min due to the breaking of the azo groups $(\mathrm{N}=\mathrm{N})$ responsible for the orange coloration, which indicated that the aromatic compounds were attacked by hydroxyl radicals forming radical intermediates (Chan et al. 2011). These experiments demonstrated the high photocatalytic efficiency of pure ZnS NCs, which generated a rapid degradation of MO molecules and the solution decolorization.

To quantify the photocatalytic activity of ZnS NCs, the degradation efficiency, degradation constant and half-life were calculated using the following equations (Dhandapani et al. 2016):

$$
\begin{gathered}
\eta=\frac{(A 0-A t)}{A 0} \times 100=\frac{(C 0-C t)}{C 0} \times 100 \\
K t=\ln \left(\frac{A_{0}}{A_{t}}\right)=\operatorname{Ln}\left(\frac{C_{0}}{C_{t}}\right) \\
t_{1 / 2}=\frac{\operatorname{Ln}(2)}{k}
\end{gathered}
$$


where $A_{0}, A_{t}, C_{0}$ and $C_{\mathrm{t}}$ are the initial absorbance, the absorbance after irradiation, the initial concentration of the solution and the concentration of the dye after irradiation for time $t$, respectively.

The values of degradation efficiency of undoped $\mathrm{ZnS}$ NCsare given in Table 5. These results revealed a high degradation efficiency of MO after just 6 min of irradiation ( 81.4\%). After 120 min of irradiation, the degradation efficiency was $98.5 \%$ and the solution became uncolored. This high efficiency may be due to the small size of the TGA molecules used as stabilizer in addition to the largest specific surface of NCs, thus generating several available adsorption sites.

The effect of manganese doping on the photocatalytic activity of MO is illustrated in Figure 13. The solution decolorization was obtained at higher contact time than that observed with the undoped ZnS NCs. Table 6 showed that doping generated animpeding effect for the MO degradation and the corresponding efficiency was lower than that observed using undoped $\mathrm{ZnS}$. The highest MO degradation efficiency was obtained for $8 \%$ manganese concentration of doped $\mathrm{ZnS}$ NCs. In terms of degradation time, this doping concentration of $\mathrm{ZnS}$ NCs required $120 \mathrm{~min}$ for obtaining an efficiency of $87.1 \%$.

The pseudo-first-order kinetic equation was used to calculate the rate constant $(k)$ for $\mathrm{MO}$ degradation using pure and Mn doped ZnS NCs where the corresponding plots of $\ln \left(A_{0} / A_{t}\right)$ versus irradiation time are given in Figure 14. The rate constants ranged from 0.281 to 0.004 $\min ^{-1}$, see Table 6 . Indeed, the trapping of charge carriers with pure $\mathrm{ZnS}$ was provided by sulfur vacancies, while multiple traps of charge carriers occurred with manganese doped $\mathrm{ZnS}$ due to structural defects in ZnS NCs (i.e., vacancies and interstices of Zn and S, interstices of $\mathrm{Mn}$ ) and other surface defects (Zong et al. 2014). These defects produced discrete energy levels within the forbidden band $\left(E_{g}\right)$ of $\mathrm{ZnS}$ NPs and, therefore, the possibility of recombination of electron and hole inside the NPs increased (Štengl et al. 2009), which resulted in a reduction of the photocatalytic activity of these NCs with low MO degradation. In view of that, it has been suggested that as the concentration of $\mathrm{Mn}^{2+}$ ions increased, a competitive process of charge carrier trapping between $\mathrm{V}_{\mathrm{S}}$ and $\mathrm{Mn}^{2+}$ occurred (Sergeeva et al. 2018).

The rate of MO degradation depended on nanocatalyst properties such as specific surface, nanocrystal size, composition, crystallinity and gap energy(Chaturvedi et al. 2012). Despite the photoluminescence, properties of $\mathrm{ZnS}$ were ameliorated via $\mathrm{Mn}^{2+}$ doping thus reaching a 
maximum for $8 \% \mathrm{ZnS}$, results showed that a low MO degradation was obtained with doped NCs. This finding can be explained by the high probability of electron-hole recombination for doped NCs and, subsequently, the strong PL response impacted indirectly on photocatalytic activity by the low density of free excitons that migrate to the surface where they generated oxidizing species(Ashkarran 2014). As a result, the presence of high photoluminescence generated a low photocatalytic activity. Consequently, undoped ZnS NCs are a promising nanocatalyst for the degradation of MO dye and its application can be extended to degrade other organic molecules.

\subsection{Toward an industrial efficient solar activated nanocatalyst}

As stated, the degradation of organic pollutants is a necessity due to environmental pollution problems. Consequently, the industries request the most efficient degradation process but with a low cost. One way to achieve this goal is the use of solar activated efficient photocatalyst. In this context, additional photodegradation experiments were carried out under solar light irradiation to evaluate the usability of undoped $\mathrm{ZnS}$ NCs for wastewater purification. Before the sunlight irradiation, the suspensions were magnetically stirred for 120 min to allow adsorption-desorption equilibrium between MO molecules and NCs. Then, the mixture was submitted to sunlight irradiation between 11 am and $2 \mathrm{pm}$ to evaluate the dye degradation. Figure 15 presents the UV-visible absorption spectra of MO degradation using $\mathrm{ZnS}$ NCs and the degradation rate as a function of time recorded after sunlight exposition. Results of Figure 15 and Table 7 showed the total decolorization of the solution after $120 \mathrm{~min}$ of sunlight exposition. This experiment confirmed the degradation efficiency of these NCs. Overall, the percentage of dye degradation increased significantly with the exposition time where $97.4 \%$ was the maximum decolorization efficiency with a degradation constant $K$ of $0.045 \mathrm{~min}^{-1}$. In fact, there was no a significant difference with the efficiency obtained under UV irradiation (98.5\%). It is convenient to note that sunlight is often exploited for thesemiconductorsirradiation with the aim of performing the photodegradation of pollutants thus contributing to reduce the cost of water treatment methods and the environmental pollution as compared to UV lamps. These results proved that undoped ZnS NCs exhibited a strong photocatalytic activity for MO dye. This behavior may be explained by the large band gap energy of $\mathrm{ZnS}$, which caused a higher redox potential of e-h pairs and, consequently, a higher photocatalytic performance (Herrmann et al. 1984). The photocatalytic efficiency of $\mathrm{ZnS}$ NCs was compared with other nanocatalysts reported in the literature and the results are summarized in Table 8. It is clear that pure $\mathrm{ZnS}$ can be a good candidate for dye degradation 
under sunlight irradiation. This material offer additional advantages such as it does not use expensive organic moieties and toxic products in the catalyst preparation together with a higher rate of decolorization under UV and sunlight irradiations(Gajendiran et al. 2020; Samanta et al. 2018; Pathania et al. 2016; Suganya et al. 2019; Samanta et al. 2020; Dake et al. 2020; Ramki et al. 2020; Matras-Postołek et al. 2019).These results are encouraging for the application of these NCs to degrade organic compounds dissolved in wastewater at great scale in the industrial sector.

\subsection{Mechanism of photocatalytic activity}

The mechanism of photocatalytic degradation of methylene orange by undoped and doped $\mathrm{ZnS}$ nanocatalysts could be explained as follows. First, UV light excitation of ZnS NCs generated the electron-hole pairs $\left(\mathrm{e}^{-} / \mathrm{h}^{+}\right)$in $\mathrm{ZnS}$. The $\left(\mathrm{OH}^{*}\right)$ radical was formed from a simple oxidation of water or hydroxyl ion $\left(\mathrm{OH}^{-}\right)$by photo-generated holes. Similarly, superoxide radicals $\left(\mathrm{O}_{2}{ }^{*}\right)$ were formed in water from a reduction reaction of the dissolved dioxygen in water by photo-generated electrons. These highly reactive hydroxyl radicals $\left(\mathrm{OH}^{*}\right)$ and superoxide radicals $\left(\mathrm{O}_{2}{ }^{*}\right)$ reacted with $\mathrm{MO}$ dye molecule adsorbed on $\mathrm{ZnS}$ nanoparticles ensuring the degradation. The next equations describe this mechanism

$$
\begin{gathered}
\mathrm{ZnS}+\mathrm{h} v \rightarrow \mathrm{eBC}^{-}+\mathrm{hBv}^{+} \\
\mathrm{e}^{-}+\mathrm{O}_{2} \rightarrow \mathrm{O}_{2}{ }^{*-} \\
\mathrm{h}^{+}+\mathrm{OH}^{-} \rightarrow \mathrm{OH}^{*} \\
\mathrm{~h}^{+}+\mathrm{H}_{2} \mathrm{O} \rightarrow \mathrm{OH}^{*}+\mathrm{H}^{+}
\end{gathered}
$$

Figure 16 summarizes the different stages of this photocatalytic process. The first step corresponded to the transfer of the MO molecule from the aqueous phase to the surface of NCs. In the second step, the organic molecules were adsorbed on the surface of the catalyst followed by the movement of photo-generated $\mathrm{e}^{-}$and $\mathrm{h}^{+}$charges to the catalyst surface. The positive hole in the NCs reacted with a water molecule to produce hydrogen gas and free hydroxyl radical $\left(\mathrm{OH}^{*}\right)$, which was the most important and powerful oxidizing radical for the mineralization of adsorbed or free MO on the catalyst surface. Photo-induced electrons were easily trapped by electronic acceptors, such as adsorbed $\mathrm{O}_{2}$, to produce a super oxide anion radical $\left(\mathrm{O}_{2}{ }^{{ }^{-}}\right)$. This reaction limited the recombination phenomena and thus improved 
degradation efficiency. Finally, these different radicals reacted with the MO to form intermediate compounds before the mineralization to $\mathrm{CO}_{2}$ and $\mathrm{H}_{2} \mathrm{O}$.

\section{Conclusions}

Nanocrystals of undoped and Mn-doped ZnS were successfully synthesized by colloidal precipitation reaction method and applied in the photodegradation of MO dye. FTIR results confirmed the functionalization of nanocrystals surface by TGA. XRD analysis indicated the formation of nanocrystals with two crystalline phases, hexagonal and cubic, identical to the solid material and with a relatively large size distribution. The average size of $\mathrm{ZnS}$ nanocrystals calculated by using Debye-Scherrer formula varied from 2.5 to $7.1 \mathrm{~nm}$. The optical absorption spectra showed the appearance of an absorption edge shifted towards short wavelengths compared to that of the solid-state semiconductor ZnS. PL spectroscopy indicated that the emission intensity increased with the doping rate up to $8 \%$ and then it decreased. Modeling results showed that the Hill model explained the adsorption mechanism of MO on the nanocrystal surface, dye removal followed a pseudo-second order reaction kinetics. Adsorption capacity of doped ZnS-TGA was $26.98 \mathrm{mg} / \mathrm{g}$ and this property contributed to the direct interaction of photo-generated electrons with adsorbed MO molecules and hence facilitated a quick photodegradation. Dye adsorption process was multimolecular via physisorption involving hydrogen bonding and van der Waals interactions. The undoped $\mathrm{ZnS}$-TGA nanocrystals exhibited high photocatalytic activity to degrade MO dye under UV and sunlight. Consequently, the pure ZnS-TGA nanocatalysts are suggested as a promising photocatalyst for wastewater purification. On the other hand, Mn-doped ZnS-TGA nanocrystals showed superior optical and physicochemical characteristics making them good candidates for fluorescence-based applications.

\section{Author's contribution:}

Sabri Ouni: Writing - original draft, Visualization, Conceptualization, Methodology

Naim Bel Haj Mohamed: Visualization, Writing - review \& editing; Conceptualization, Methodology

Noureddine Chaaben: Resources, Writing - review \& editing Adrian Bonilla-Petriciolet:Writing - Reviewing and Editing Mohamed Haouari:Writing - Reviewing and Editing, Visualization, Supervision 
559

560

561

562

563

564

565

566

567

568

569

570

571

572

573

574

575

576

577

578

579

580

581

582

583

584

585

586

587

588

589

590

591

592

\section{Funding:}

The authors declare that they have no known competing financial interests or personal relationships that could have appeared to influence the work reported in this paper.

Availability of data and materials: Data and materials are contained within the article

\section{Declarations}

Ethics approval and consent to participate: Not applicable for this study.

Consent to publish: All authors consent to this publication.

Competing interests: The authors declare no competing interests

(1)

.




\section{References}

595

596

597

598

599

600

601

602

603

604

605

606

607

608

609

610

611

612

613

614

615

616

617

618

619

620

621

622

623

624

625

626

627

628

629

630
Ajibade PA, Oluwalana AE, Sikakane BM, Singh M (2020) Structural, photocatalytic and anticancer studies of hexadecylamine capped ZnS nanoparticles. Chem Phys Lett 755:137813. https://doi.org/10.1016/j.cplett.2020.137813

Amaranatha Reddy D, Liu C, Vijayalakshmi RP, Reddy BK (2014) Effect of Al doping on the structural, optical and photoluminescence properties of ZnS nanoparticles. J Alloys Compd 582:257-264. https://doi.org/10.1016/j.jallcom.2013.08.051

Amirian F, Molaei M, Karimipour M, Bahador AR (2018) A new and simple UV-assisted approach for synthesis of water soluble ZnS and transition metals doped ZnS nanoparticles (NPs) and investigating optical and photocatalyst properties. J Lumin 196:174-180.

https://doi.org/10.1016/j.jlumin.2017.12.005

Arao Y, Hirooka Y, Tsuchiya K, Mori Y (2009) Structure and Photoluminescence Properties of Zinc Sulfide Nanoparticles Prepared in a Clay Suspension. J Phys Chem C 113:894-899.

https://doi.org/10.1021/jp8051449

Ashkarran AA (2014) Absence of photocatalytic activity in the presence of the photoluminescence property of $\mathrm{Mn}-\mathrm{ZnS}$ nanoparticles prepared by a facile wet chemical method at room temperature. Mater SciSemicond Process 17:1-6. https://doi.org/10.1016/j.mssp.2013.08.003

Atrous M, Sellaoui L, Bouzid M, et al (2019) Adsorption of dyes acid red 1 and acid green 25 on grafted clay: Modeling and statistical physics interpretation. J Mol Liq 294:111610.

https://doi.org/10.1016/j.molliq.2019.111610

Bel Haj Mohamed N, Bouzidi M, Ben brahim N, et al (2021) Impact of the stacking fault and surface defects states of colloidal CdSe nanocrystals on the removal of reactive black 5. Mater SciEng $B$ 265:115029. https://doi.org/10.1016/j.mseb.2020.115029

Bouzid M, Bouaziz N, Torkia YB, Lamine AB (2019) Statistical physics modeling of ethanol adsorption onto the phenol resin based adsorbents: Stereographic, energetic and thermodynamic investigations. J Mol Liq 283:674-687. https://doi.org/10.1016/j.molliq.2019.03.129

Bouzid M, Sellaoui L, Khalfaoui M, et al (2016) Adsorption of ethanol onto activated carbon:

Modeling and consequent interpretations based on statistical physics treatment. Phys Stat Mech Its Appl 444:853-869. https://doi.org/10.1016/j.physa.2015.09.097

Brus L (1986) Electronic wave functions in semiconductor clusters: experiment and theory. J Phys Chem 90:2555-2560. https://doi.org/10.1021/j100403a003

Bujňáková Z, Dutková E, Kello M, et al (2017) Mechanochemistry of Chitosan-Coated Zinc Sulfide (ZnS) Nanocrystals for Bio-imaging Applications. Nanoscale Res Lett 12:328.

https://doi.org/10.1186/s11671-017-2103-z

Buthiyappan A, Aziz ARA, Daud WMAW (2016) Recent advances and prospects of catalytic advanced oxidation process in treating textile effluents. Rev ChemEng 32:1-47. https://doi.org/10.1515/revce2015-0034 
Chan SHS, Wu TY, Juan JC, Teh CY (2011) Recent developments of metal oxide semiconductors as photocatalysts in advanced oxidation processes (AOPs) for treatment of dye waste-water. J ChemTechnolBiotechnol 86:1130-1158. https://doi.org/10.1002/jctb.2636

Chang L, He X, Chen L, Zhang Y (2017) Mercaptophenylboronic acid-capped Mn-doped ZnS quantum dots for highly selective and sensitive fluorescence detection of glycoproteins. Sens Actuators B Chem 243:72-77. https://doi.org/10.1016/j.snb.2016.11.121

Chaturvedi S, Dave PN, Shah NK (2012) Applications of nano-catalyst in new era. J Saudi ChemSoc 16:307-325. https://doi.org/10.1016/j.jscs.2011.01.015

Chauhan R, Kumar A, Pal Chaudhary R (2014) Photocatalytic degradation of methylene blue with Cu doped ZnS nanoparticles. J Lumin 145:6-12. https://doi.org/10.1016/j.jlumin.2013.07.005

Dake DV, Raskar ND, Mane VA, et al (2020) Exploring the role of defects on diverse properties of Crsubstituted ZnS nanostructures for photocatalytic applications. Appl Phys A 126:640. https://doi.org/10.1007/s00339-020-03669-1

Dao V-D (2020) Bimetallic PtSe nanoparticles incorporating with reduced graphene oxide as efficient and durable electrode materials for liquid-junction photovoltaic devices. Mater Today Energy 16:100384. https://doi.org/10.1016/j.mtener.2020.100384

Daskalakis I, Vamvasakis I, T. Papadas I, et al (2020) Surface defect engineering of mesoporous $\mathrm{Cu} / \mathrm{ZnS}$ nanocrystal-linked networks for improved visible-light photocatalytic hydrogen production. InorgChem Front 7:4687-4700. https://doi.org/10.1039/D0QI01013H

Dehghan F, Molaei M, Amirian F, et al (2018) Improvement of the optical and photocatalytic properties of ZnSe QDs by growth of ZnS shell using a new approach. Mater Chem Phys 206:76-84. https://doi.org/10.1016/j.matchemphys.2017.11.061

Dhandapani C, Narayanasamy R, Karthick SN, et al (2016) Drastic photocatalytic degradation of methylene blue dye by neodymium doped zirconium oxide as photocatalyst under visible light irradiation. Optik 127:10288-10296. https://doi.org/10.1016/j.ijleo.2016.08.048

Fang Z, Wu P, Zhong X, Yang Y-J (2010) Synthesis of highly luminescent Mn:ZnSe/ZnS nanocrystals in aqueous media. Nanotechnology 21:305604. https://doi.org/10.1088/0957-4484/21/30/305604

Gaceur M, Giraud M, Hemadi M, et al (2012) Polyol-synthesized Zn0.9Mn0.1S nanoparticles as potential luminescent and magnetic bimodal imaging probes: synthesis, characterization, and toxicity study. J Nanoparticle Res 14:932. https://doi.org/10.1007/s11051-012-0932-3

Gajendiran J, Gnanam S, Vijaya Kumar V, et al (2020) Structural, optical and photocatalytic properties of ZnS spherical/flake nanostructures by sugar-assisted hydrothermal process. Chem Phys Lett 754:137639. https://doi.org/10.1016/j.cplett.2020.137639

Ghasemi M, Ghasemi N, Zahedi G, et al (2014) Kinetic and equilibrium study of Ni(II) sorption from aqueous solutions onto Peganumharmala-L. Int J Environ SciTechnol 11:1835-1844. https://doi.org/10.1007/s13762-014-0617-9

Herrmann J-M, Disdier J, Pichat P (1984) Effect of chromium doping on the electrical and catalytic properties of powder titania under UV and visible illumination. Chem Phys Lett 108:618-622. https://doi.org/10.1016/0009-2614(84)85067-8 
Ho YS, McKay G (1999) Pseudo-second order model for sorption processes. Process Biochem 34:451465. https://doi.org/10.1016/S0032-9592(98)00112-5

Jai Kumar B, Mahesh HM (2017) Concentration-dependent optical properties of TGA stabilized CdTe Quantum dots synthesized via the single injection hydrothermal method in the ambient environment. SuperlatticesMicrostruct 104:118-127. https://doi.org/10.1016/j.spmi.2017.02.023

Jaldurgam FF, Ahmad Z, Touati F (2021) Synthesis and Performance of Large-Scale Cost-Effective Environment-Friendly Nanostructured Thermoelectric Materials. Nanomaterials 11:1091. https://doi.org/10.3390/nano11051091

Jothibas M, Manoharan C, Johnson Jeyakumar S, et al (2018) Synthesis and enhanced photocatalytic property of Ni doped ZnS nanoparticles. Sol Energy 159:434-443. https://doi.org/10.1016/j.solener.2017.10.055

Jothibas M, Manoharan C, Johnson Jeyakumar S, et al (2016) Photocatalytic activity of spray deposited ZrO2 nano-thin films on methylene blue decolouration. J Mater Sci Mater Electron 27:5851-5859. https://doi.org/10.1007/s10854-016-4502-9

Kabachii YA, KochevSYu, AntonovaOYu, et al (2021) Mn2+-doped ZnS-CdS alloy nanocrystals for the photocatalytic hydrogen evolution reaction. Mendeleev Commun 31:315-318.

https://doi.org/10.1016/j.mencom.2021.04.012

Kaur J, Sharma M, Pandey OP (2015) Photoluminescence and photocatalytic studies of metal ions (Mn and Ni) doped ZnS nanoparticles. Opt Mater 47:7-17.

https://doi.org/10.1016/j.optmat.2015.06.022

Kolmykov O, Coulon J, Lalevée J, et al (2014) Aqueous synthesis of highly luminescent glutathionecapped Mn2+-doped ZnS quantum dots. Mater SciEng C 44:17-23.

https://doi.org/10.1016/j.msec.2014.07.064

Kostrov AN, Gostev FE, Shelaev IV, et al (2020) Relaxation Dynamics of Excited States in Mn2+-Doped ZnCdS (Core)/ZnS (Shell) Quantum Dots Ions in Propylene Carbonate. High Energy Chem 54:421-426. https://doi.org/10.1134/S0018143920060090

Li W, Ju B, Zhang S (2019) A green I -cysteine modified cellulose nanocrystals biosorbent for adsorption of mercury ions from aqueous solutions. RSC Adv 9:6986-6994.

https://doi.org/10.1039/C9RA00048H

Liu I-S, Lo H-H, Chien C-T, et al (2008) Enhancing photoluminescence quenching and photoelectric properties of $\mathrm{CdSe}$ quantum dots with hole accepting ligands. J Mater Chem 18:675-682. https://doi.org/10.1039/B715253A

Long F, Ning S, Zhang W, et al (2020) All-fiber passively Q-switched erbium-doped laser with Cr2\$ \mathplus\$:ZnSe nanocrystals saturable absorber. Laser Phys 30:055101. https://doi.org/10.1088/1555-6611/ab8233

Mansur HS, Mansur AAP (2011) CdSe quantum dots stabilized by carboxylic-functionalized PVA: Synthesis and UV-vis spectroscopy characterization. Mater Chem Phys 125:709-717. https://doi.org/10.1016/j.matchemphys.2010.09.068

Matras-Postołek K, Sovinska S, Węgrzynowicz A (2019) Synthesis and characterization of ZnSe and ZnSe:Mnnanosheets and microflowers with high photoactive properties by microwave-assisted 
method. ChemEng Process - Process Intensif 135:204-216.

https://doi.org/10.1016/j.cep.2018.11.022

Meikle TG, Dyett BP, Strachan JB, et al (2020) Preparation, Characterization, and Antimicrobial Activity of Cubosome Encapsulated Metal Nanocrystals. ACS Appl Mater Interfaces 12:6944-6954. https://doi.org/10.1021/acsami.9b21783

Meng L, Maçarico A, Martins R (1995) Study of annealed indium tin oxide films prepared by $\mathrm{rf}$ reactive magnetron sputtering. Vacuum 46:673-680. https://doi.org/10.1016/0042-207X(94)00150-2

Mohagheghpour E, Rabiee M, Moztarzadeh F, et al (2009) Controllable synthesis, characterization and optical properties of ZnS:Mn nanoparticles as a novel biosensor. Mater SciEng C 29:1842-1848. https://doi.org/10.1016/j.msec.2009.02.012

Mohamed B, Qingyu Z, D. Moggridge G, Abdelmottaleb BL (2018) New insight in adsorption of pyridine on the two modified adsorbents types MN200 and MN500 by means of grand canonical ensemble. J Mol Liq 263:413-421. https://doi.org/10.1016/j.molliq.2018.05.008

Mote VD, Purushotham Y, Dole BN (2013) Structural, morphological and optical properties of Mn doped ZnS nanocrystals. 6

Nath D, Singh F, Das R (2020) X-ray diffraction analysis by Williamson-Hall, Halder-Wagner and sizestrain plot methods of CdSe nanoparticles- a comparative study. Mater Chem Phys 239:122021. https://doi.org/10.1016/j.matchemphys.2019.122021

Othman AA, Osman MA, Ali MA, et al (2020) Sonochemically synthesized Ni-doped ZnS nanoparticles: structural, optical, and photocatalytic properties. J Mater Sci Mater Electron 31:1752-1767. https://doi.org/10.1007/s10854-019-02693-z

Ouni S, Bel Haj Mohamed N, Bouzidi M, et al (2021) High impact of thiol capped ZnS nanocrystals on the degradation of single and binary aqueous solutions of industrial azo dyes under sunlight. J Environ ChemEng 9:105915. https://doi.org/10.1016/j.jece.2021.105915

Pan B, Sun K, Xing B (2010) Adsorption kinetics of $17 \alpha$-ethinyl estradiol and bisphenol A on carbon nanomaterials. II. Concentration-dependence. J SoilsSediments 10:845-854.

https://doi.org/10.1007/s11368-009-0185-7

Pandya SG, Corbett JP, Jadwisienczak WM, Kordesch ME (2016) Structural characterization and X-ray analysis by Williamson-Hall method for Erbium doped Aluminum Nitride nanoparticles, synthesized using inert gas condensation technique. Phys E Low-DimensSyst Nanostructures 79:98-102. https://doi.org/10.1016/j.physe.2015.12.013

Pang X, Bouzid M, dos Santos JMN, et al (2020) Theoretical study of indigotine blue dye adsorption on CoFe2O4/chitosan magnetic composite via analytical model. Colloids Surf PhysicochemEng Asp 589:124467. https://doi.org/10.1016/j.colsurfa.2020.124467

Pathania D, Gupta D, Al-Muhtaseb AH, et al (2016) Photocatalytic degradation of highly toxic dyes using chitosan-g-poly(acrylamide)/ZnS in presence of solar irradiation. J Photochem Photobiol Chem 329:61-68. https://doi.org/10.1016/j.jphotochem.2016.06.019

Pejjai B, Minnam Reddy VR, Seku K, et al (2017) Chemical bath deposition of Mn-doped ZnS thin films using greener complexing agents: Effect of Mn-doping on the optical properties. Optik 130:608-618. https://doi.org/10.1016/j.ijleo.2016.10.083 
Peng WQ, Cong GW, Qu SC, Wang ZG (2006) Synthesis and photoluminescence of ZnS:Cu nanoparticles. Opt Mater 29:313-317. https://doi.org/10.1016/j.optmat.2005.10.003

Phuong Nguyen T, Duy Le A, Bich Vu T, Vinh Lam Q (2017) Investigations on photoluminescence enhancement of poly(vinylalcohol)-encapsulated Mn-doped ZnS quantum dots. J Lumin 192:166172. https://doi.org/10.1016/j.jlumin.2017.06.031

Poornaprakash B, Chalapathi U, Suh Y, et al (2018) Terbium-doped ZnS quantum dots: Structural, morphological, optical, photoluminescence, and photocatalyticproperties. Ceram Int 44:1172411729. https://doi.org/10.1016/j.ceramint.2018.03.250

Ramki K, RajaPriya A, Sakthivel P, et al (2020) Rapid degradation of organic dyes under sunlight using tin-doped ZnS nanoparticles. J Mater Sci Mater Electron 31:8750-8760.

https://doi.org/10.1007/s10854-020-03410-x

Rtimi S, Kiwi J, Nadtochenko V (2021) Photo-induced environmental remediation, biomedical imaging, and microbial inactivation by Mn-doped semiconductors: critical issues. CurrOpinChemEng 34:100731. https://doi.org/10.1016/j.coche.2021.100731

\section{S L (1898) ABOUT THE THEORY OF SO-CALLED ADSORPTION OF SOLUBLE SUBSTANCES. 24:1-39}

Saenger DU, Jung G, Menning M (1998) Optical and Structural Properties of Doped ZnS Nanoparticles Produced by the Sol-Gel Method. J Sol-Gel SciTechnol 13:635-639.

https://doi.org/10.1023/A:1008663831823

Samanta D, Basnet P, InakhunbiChanu T, Chatterjee S (2020) Biomolecule assisted morphologycontrollable synthesis of Zinc Sulphide nanomaterials for efficient photocatalytic activity under solar irradiation. J Alloys Compd 844:155810. https://doi.org/10.1016/j.jallcom.2020.155810

Samanta D, Chanu TI, Basnet P, Chatterjee S (2018) Organic Dye Degradation Under Solar Irradiation by Hydrothermally Synthesized ZnS Nanospheres. J Mater Eng Perform 27:2673-2678. https://doi.org/10.1007/s11665-018-3214-0

Sergeeva KA, Sergeev AA, Kuznetsova YV, Voznesenskiy SS (2018) ZnS:Mn2+ Quantum Dots as Efficient Photocatalyst for Organic Dye Decolorization. Defect Diffus Forum 386:207-213. https://doi.org/10.4028/www.scientific.net/DDF.386.207

Sghaier W, Ben Torkia Y, Bouzid M, Ben Lamine A (2021) CO2 adsorption investigation by statistical physics: Thermodynamic analysis for cooling cycle application. J Environ ChemEng 9:105108. https://doi.org/10.1016/j.jece.2021.105108

Singh V, Chauhan P (2009) Structural and optical characterization of CdS nanoparticles prepared by chemical precipitation method. J Phys Chem Solids 70:1074-1079.

https://doi.org/10.1016/j.jpcs.2009.05.024

Štengl V, Bakardjieva S, Murafa N (2009) Preparation and photocatalytic activity of rare earth doped TiO2 nanoparticles. Mater Chem Phys 114:217-226.

https://doi.org/10.1016/j.matchemphys.2008.09.025

Su D, Wang L, Li M, et al (2020) Highly luminescent water-soluble AgInS2/ZnS quantum dots-hydrogel composites for warm white LEDs. J Alloys Compd 824:153896.

https://doi.org/10.1016/j.jallcom.2020.153896 
Suganya S, Jothibas M, Jeyakumar SJ (2019) Solid state synthesis of cadmium doped ZnS with 790 excellent photocatalytic activity and enhanced visible light emission. J Mater Sci Mater Electron 791 30:7916-7927. https://doi.org/10.1007/s10854-019-01113-6

792 Tuan NT, Trung DQ, Quang NV, et al (2018) Excitation energy dependence of the life time of orange 793 emission from Mn-doped ZnS nanocrystals. J Lumin 199:39-44.

794 https://doi.org/10.1016/j.jlumin.2018.02.062

795 Wang L, Wang P, Huang B, et al (2017) Synthesis of Mn-doped ZnS microspheres with enhanced 796 visible light photocatalytic activity. Appl Surf Sci 391:557-564.

797 https://doi.org/10.1016/j.apsusc.2016.06.159

798 Wang X, Shi J, Feng Z, et al (2011) Visible emission characteristics from different defects of ZnS

799 nanocrystals. Phys ChemChem Phys 13:4715-4723. https://doi.org/10.1039/COCP01620A

800 Wieszczycka K, Staszak K, Woźniak-Budych MJ, et al (2021) Surface functionalization - The way for 801 advanced applications of smart materials. CoordChem Rev 436:213846.

802 https://doi.org/10.1016/j.ccr.2021.213846

803 Xie R, Li L, Li Y, et al (2011) Fe:ZnSe semiconductor nanocrystals: Synthesis, surface capping, and 804 optical properties. J Alloys Compd 509:3314-3318. https://doi.org/10.1016/j.jallcom.2010.12.046

805 Yang L, Zhou H, Fan T, Zhang D (2014) Semiconductor photocatalysts for water oxidation: current 806 status and challenges. Phys ChemChem Phys 16:6810-6826. https://doi.org/10.1039/C4CP00246F

807 Zong Y, Li Z, Wang X, et al (2014) Synthesis and high photocatalytic activity of Eu-doped ZnO 808 nanoparticles. Ceram Int 40:10375-10382. https://doi.org/10.1016/j.ceramint.2014.02.123 809 


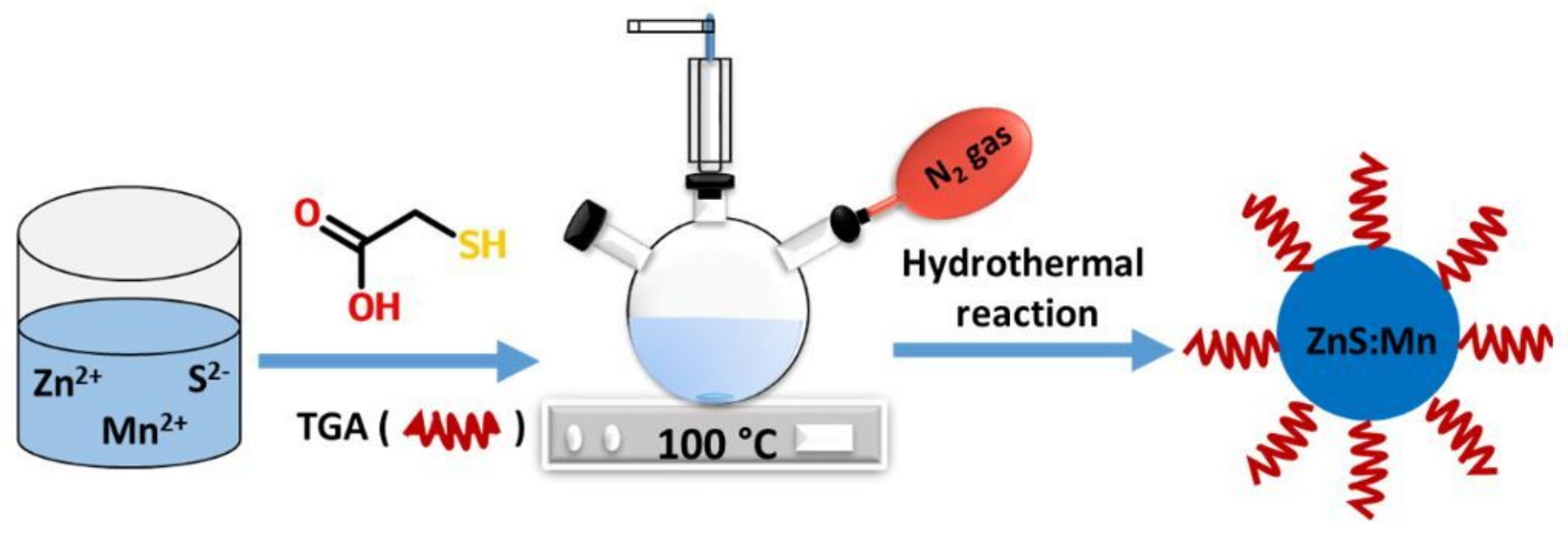

Figure 1

Description of synthesis protocol to obtain Mn-doped ZnS-TGA nanocrystals.
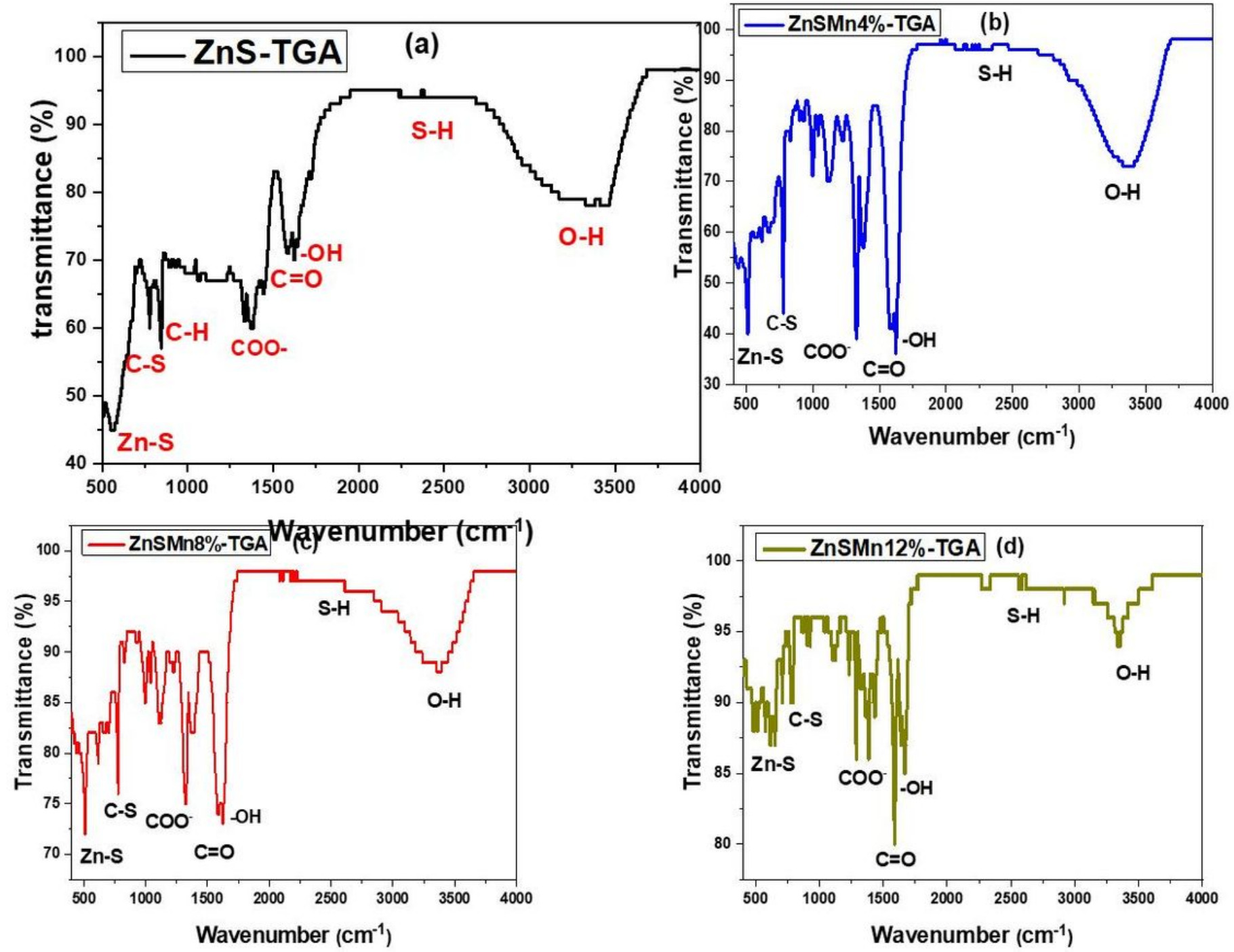
Figure 2

FTIR spectra of (a) undoped and Mn-doped ZnS-TGAnanocrystals with doping of (b) $4 \%$, (c) $8 \%$ and (d) $12 \%$.
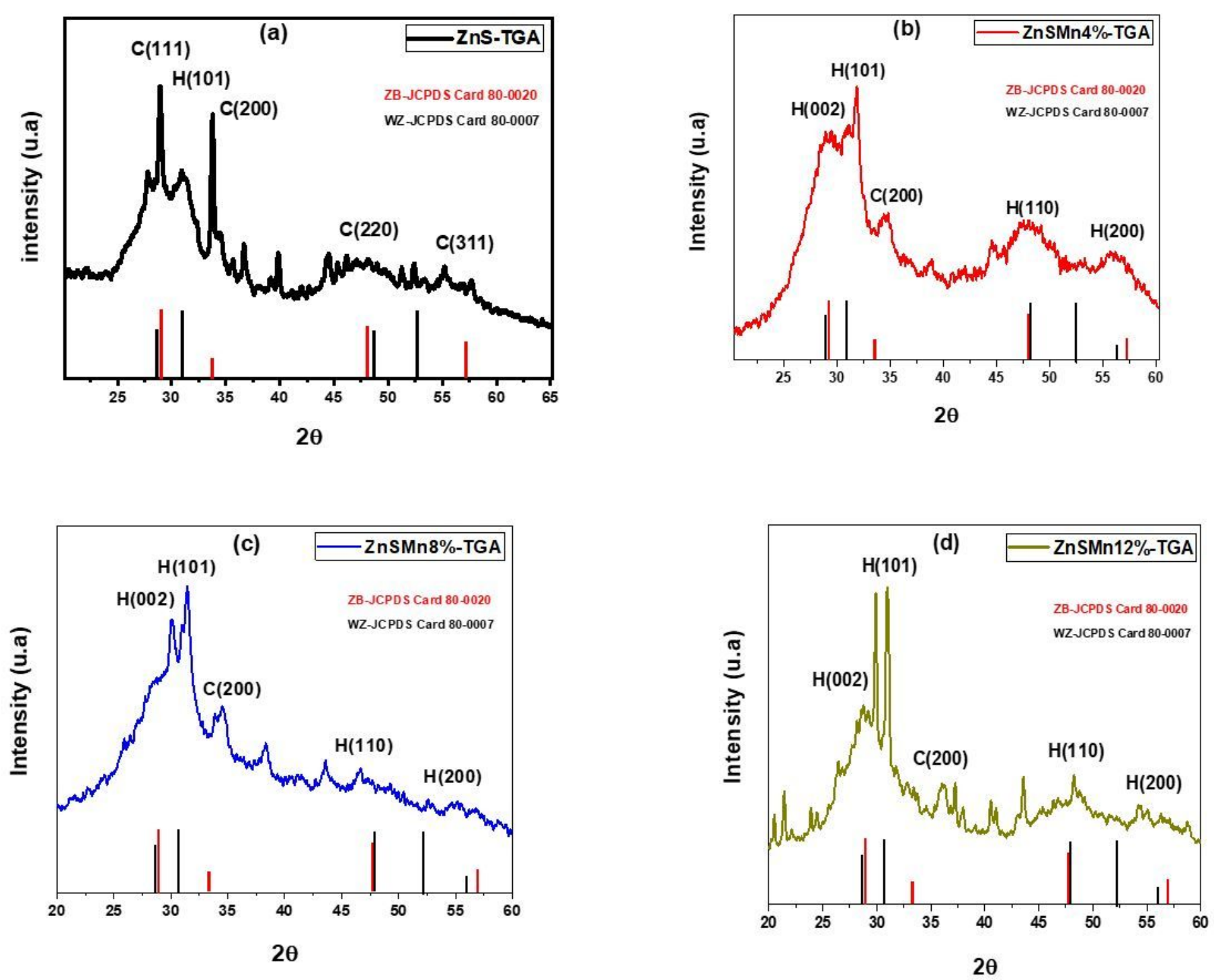

Figure 3

DRX patterns of undoped and Mn-doped ZnS-TGAnanocrystals. 

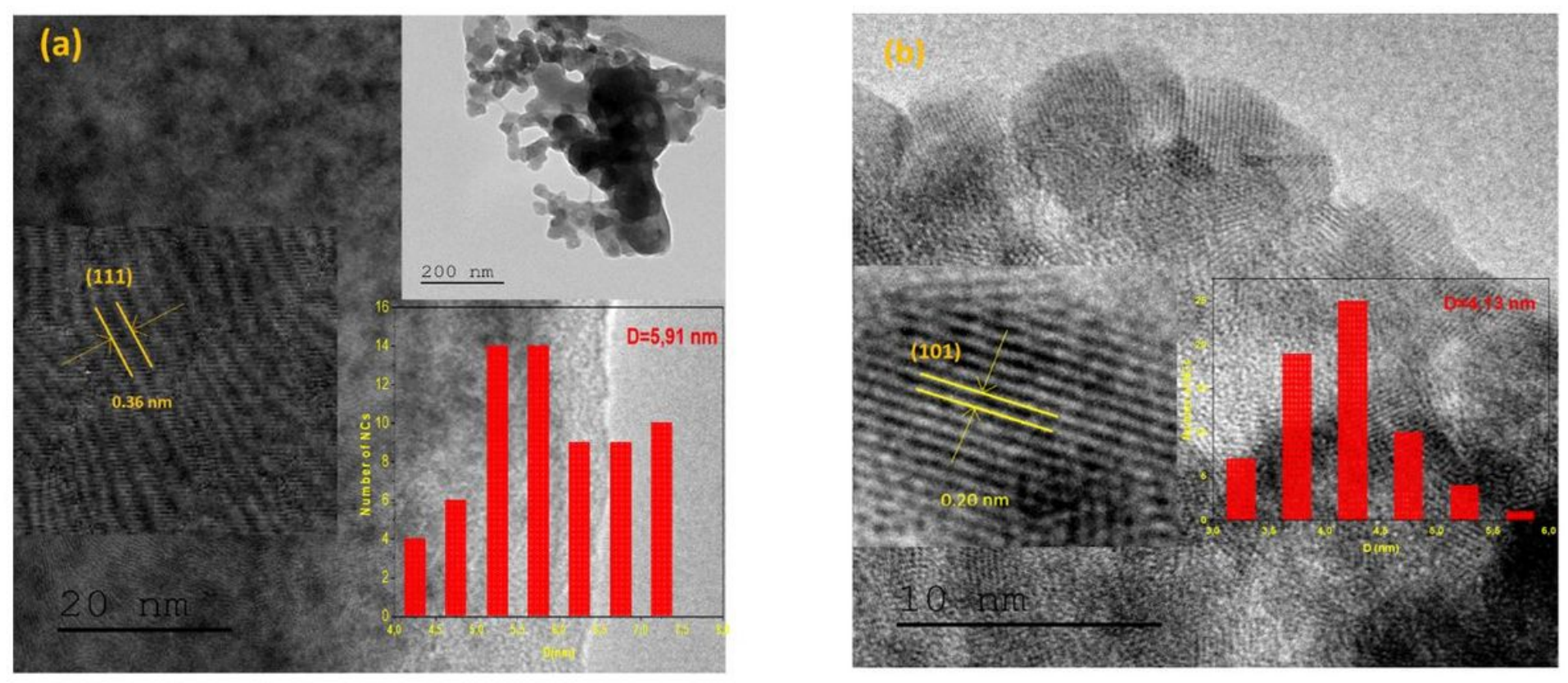

Figure 4

TEM images of (a) undoped and (b) Mn-doped ZnS-TGA nanocrystals. 

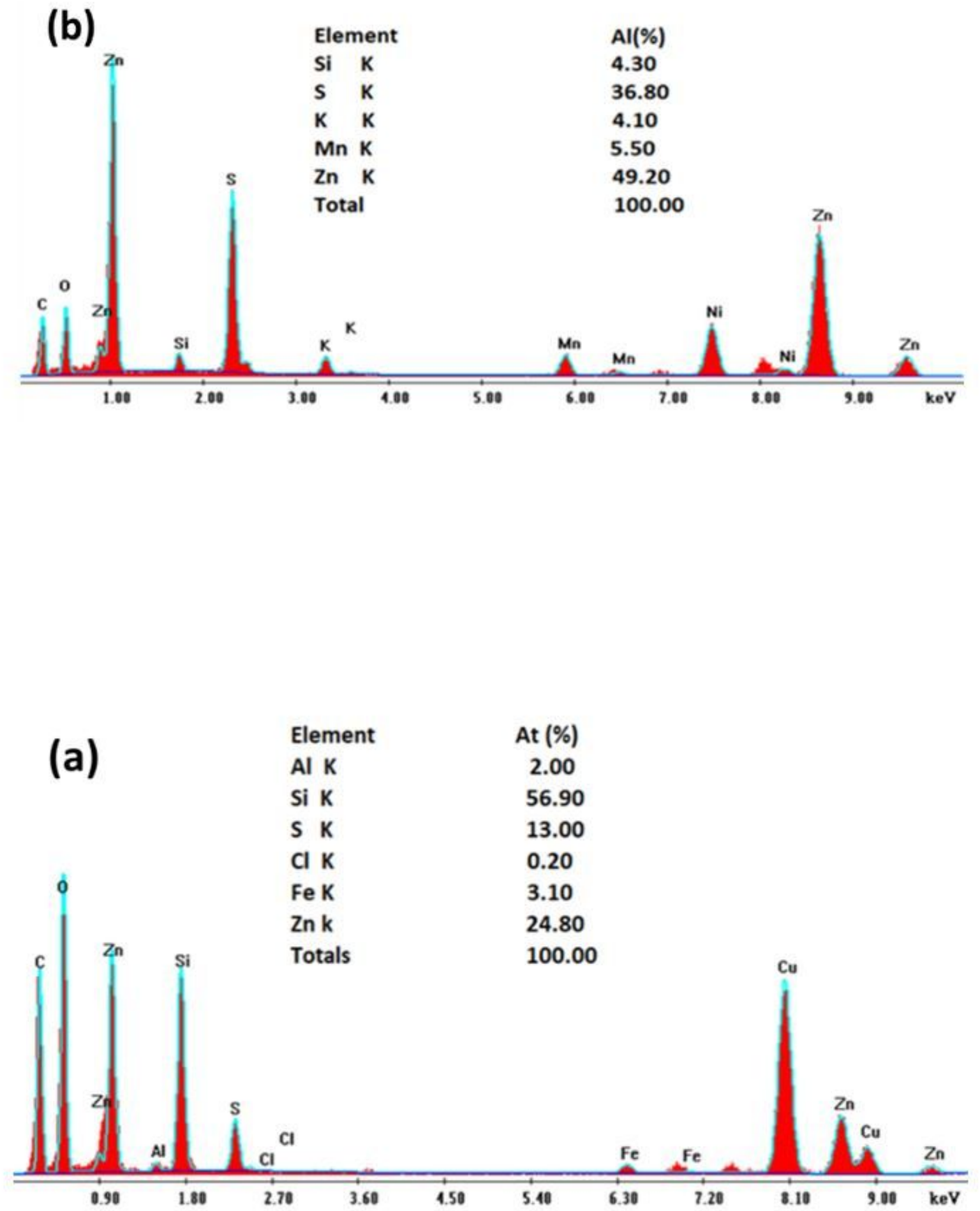

Figure 5

EDX spectrum of (a) undoped and (b) Mn-doped ZnS-TGAnanocrystals. 


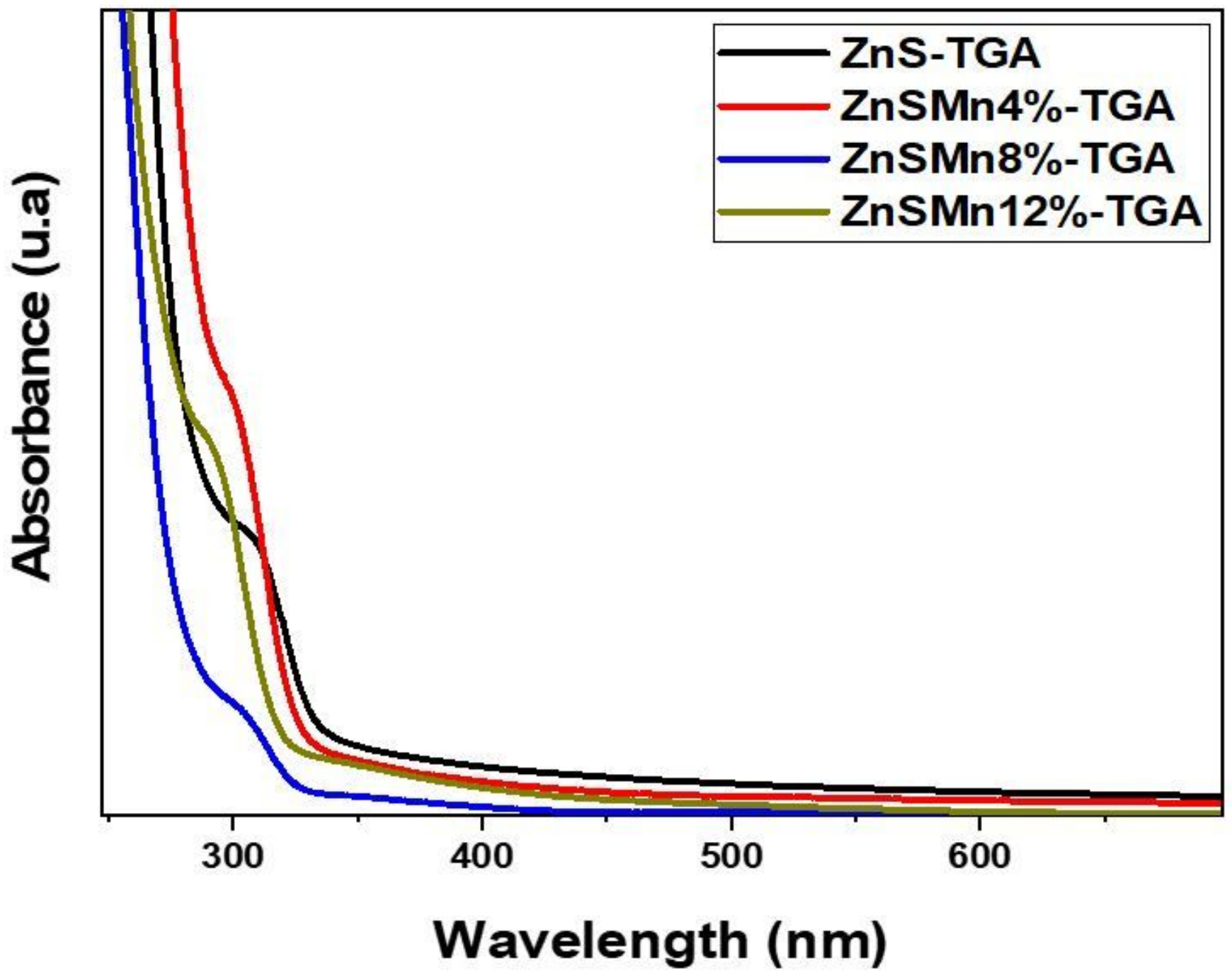

Figure 6

Optical absorption spectra of undoped and Mn-doped ZnS-TGA nanocrystals. 

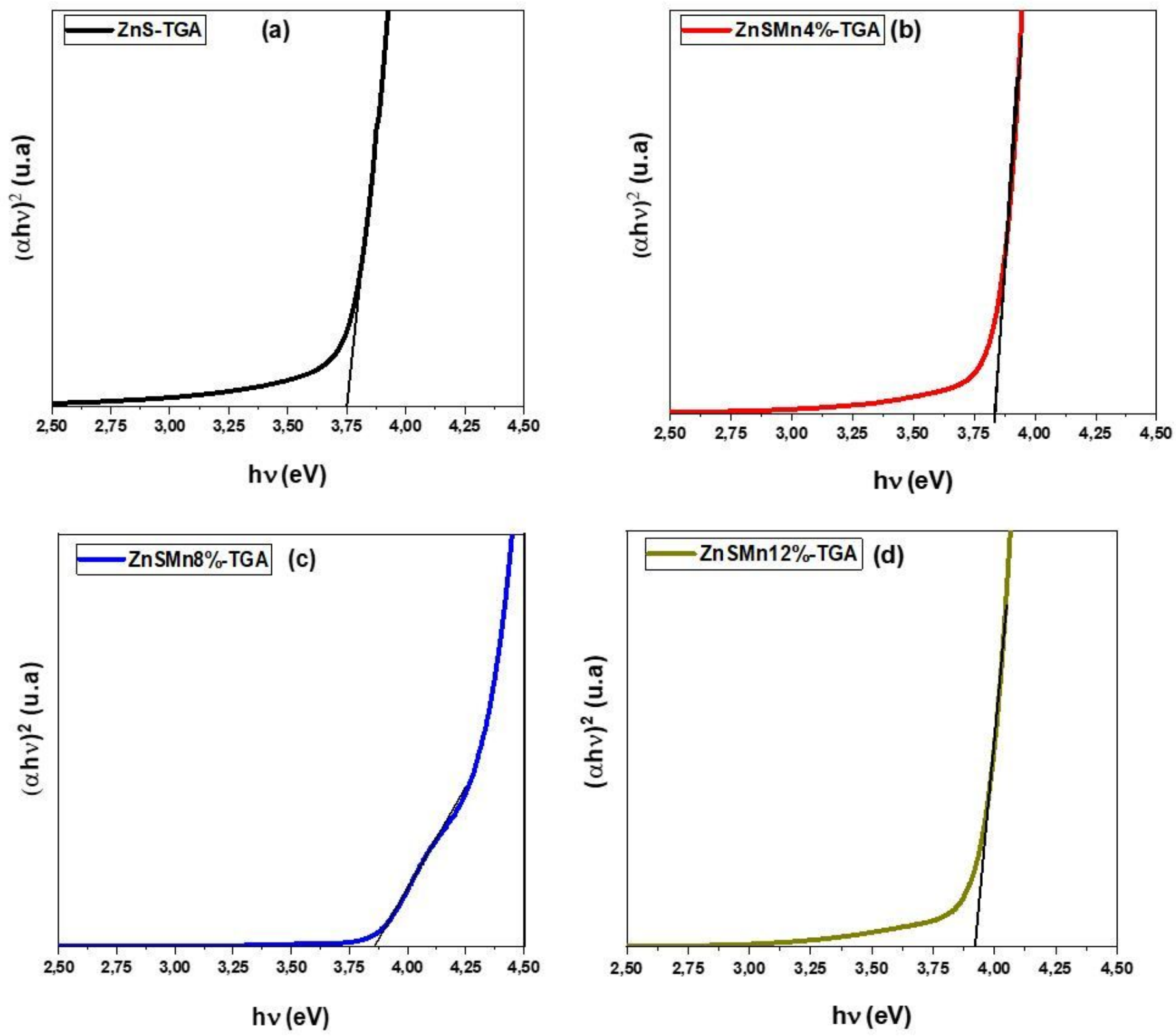

Figure 7

Graphical method for the calculation of the optical gap of (a) undoped and (b-d) Mn-doped ZnS-TGA nanocrystals. 


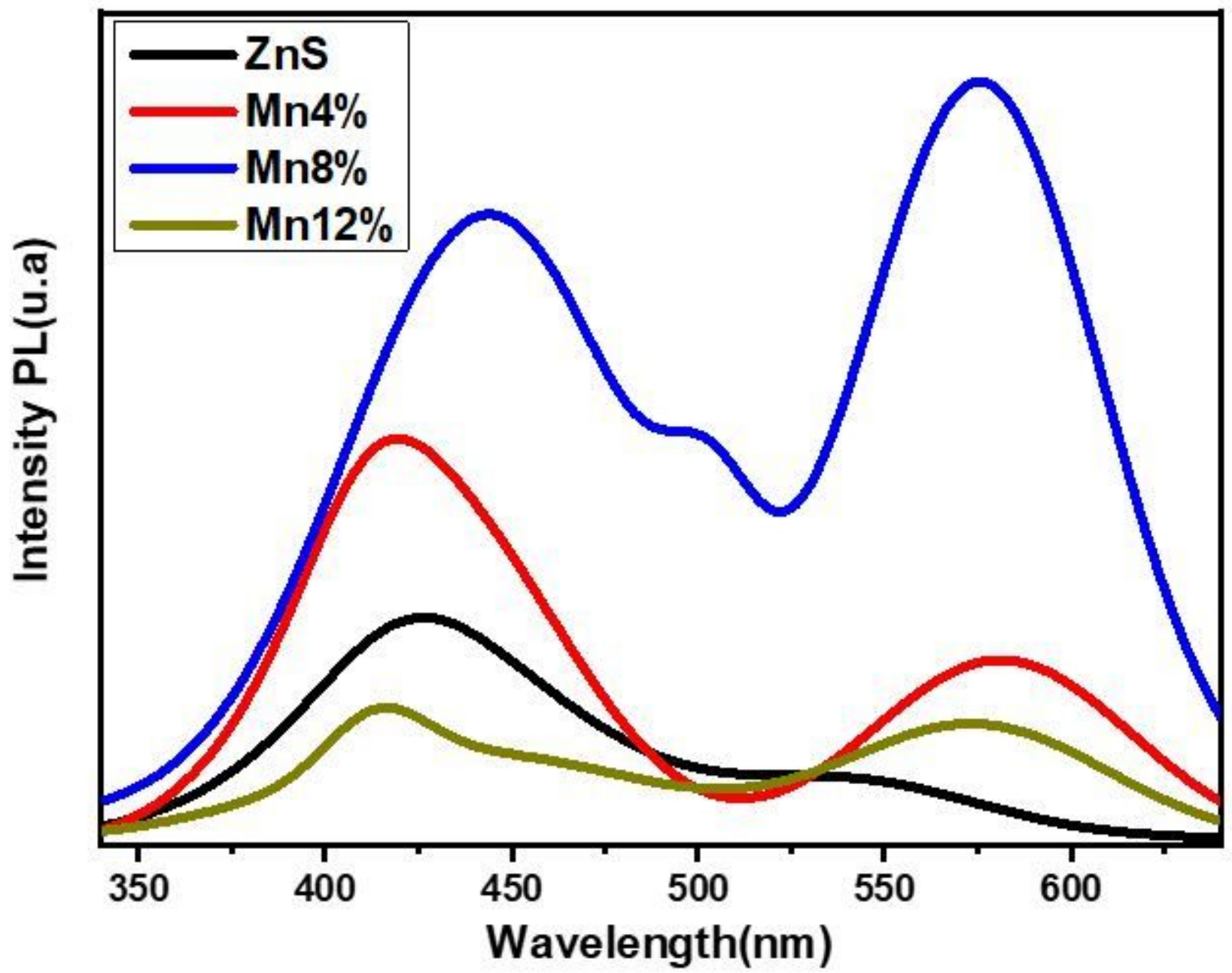

Figure 8

Emission spectra of undoped and Mn-doped ZnS-TGA nanocrystals. 

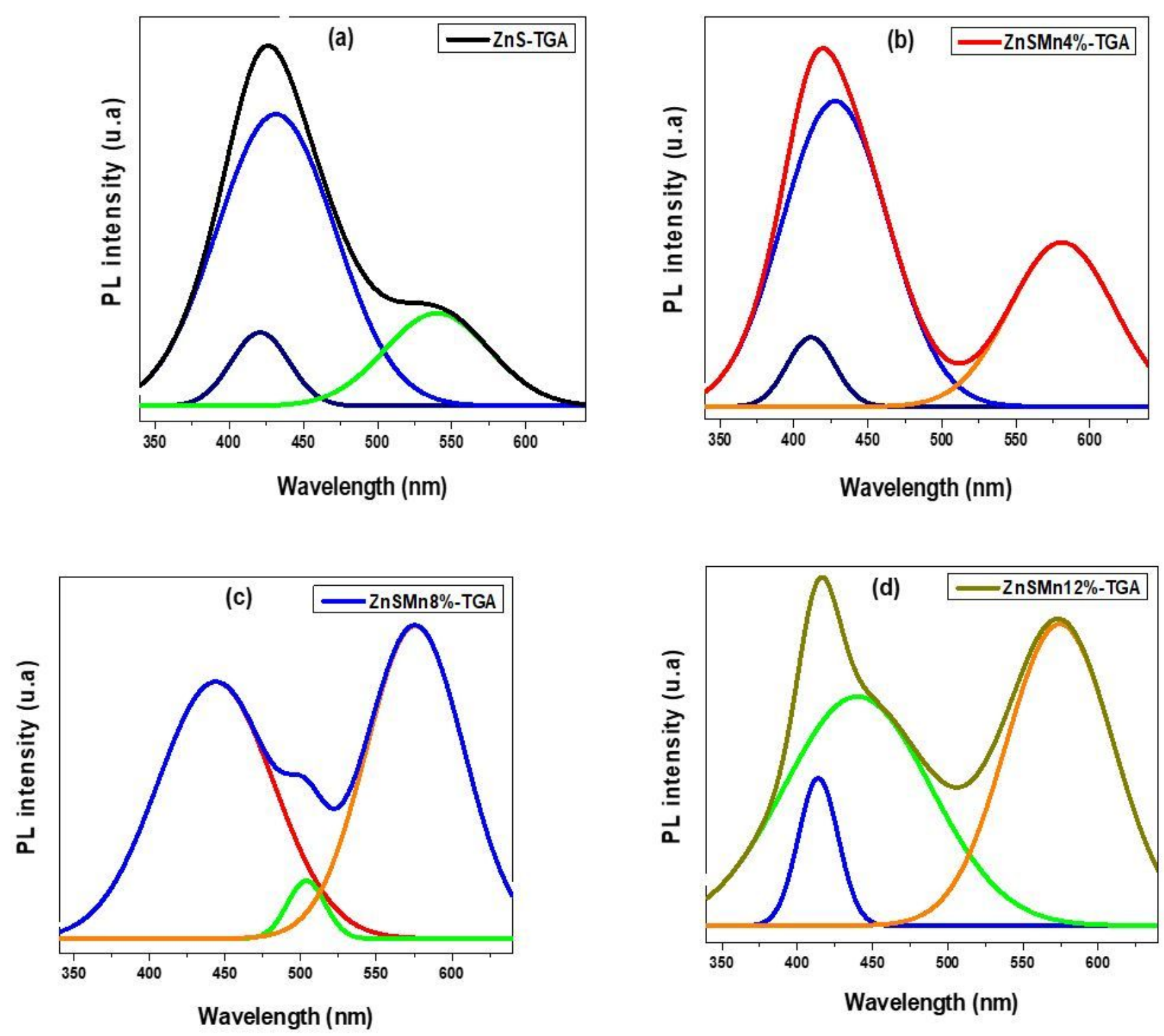

Figure 9

Gaussian adjustment of PL spectra of (a) undoped and (b-d) Mn-doped ZnS-TGA nanocrystals. 


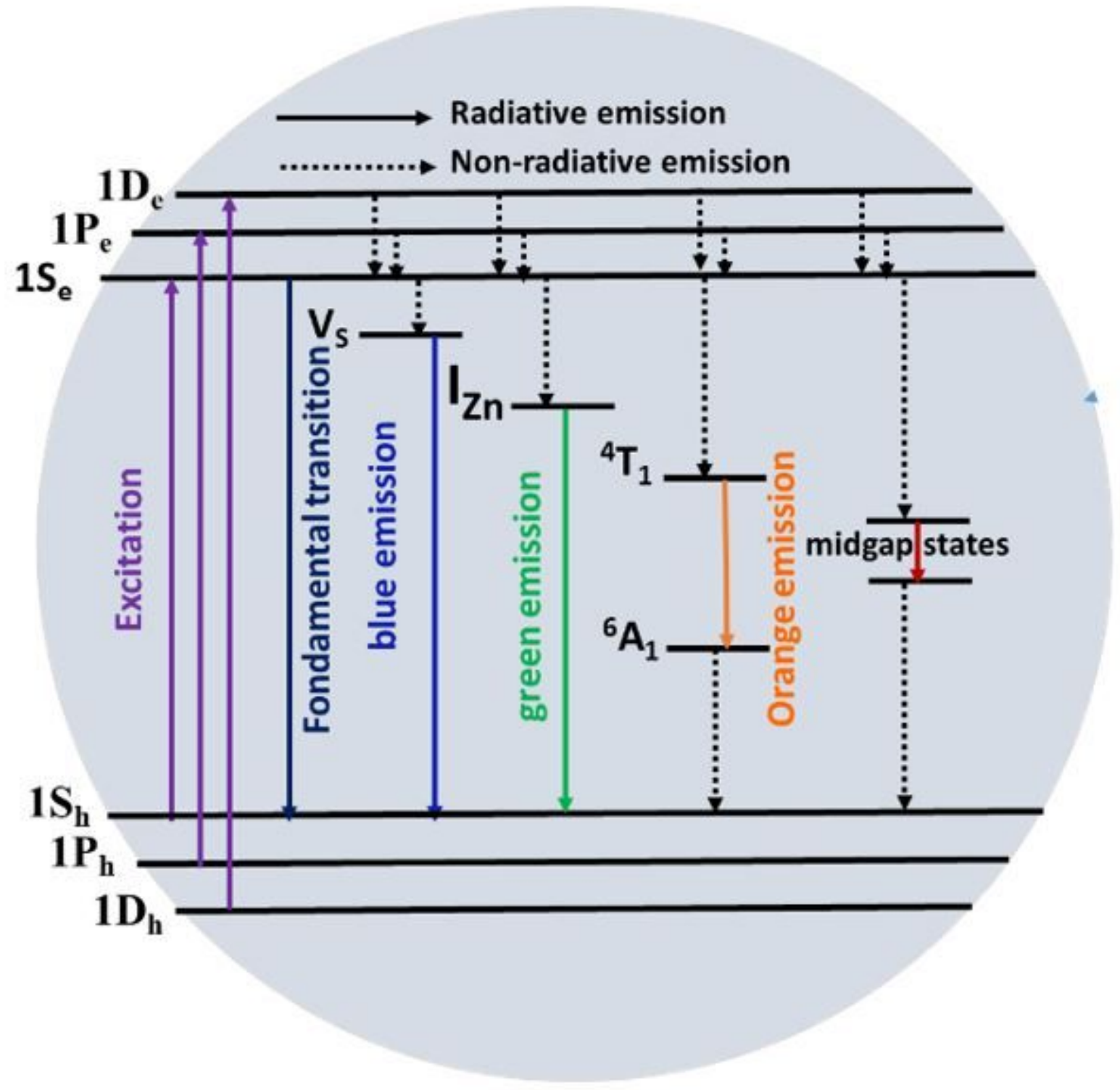

Figure 10

Illustration of emission mechanism of Mn-doped ZnS-TGA nanocrystals. 


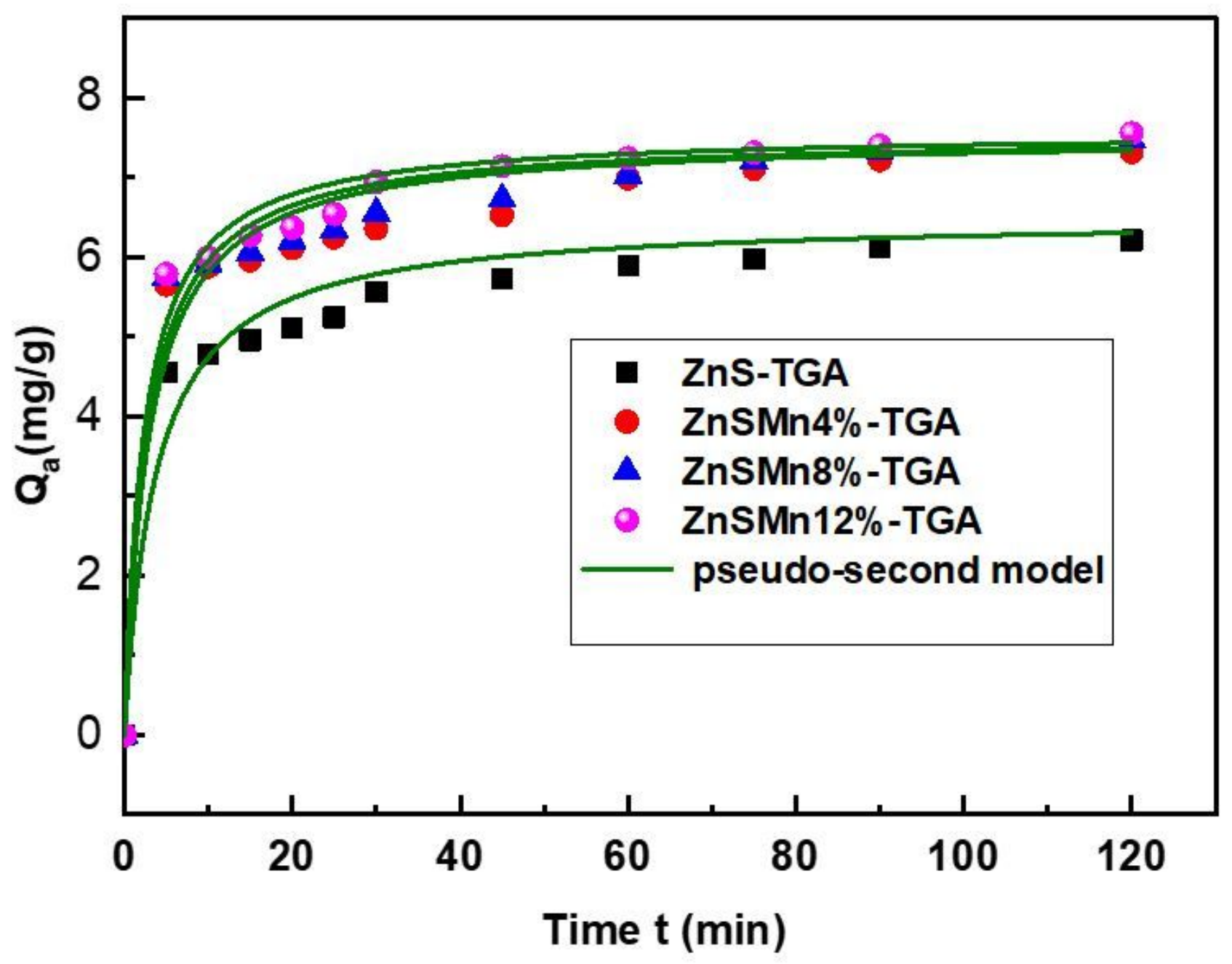

Figure 11

Kinetic adsorption of MO on Mn-doped ZnS-TGA nanocrystals. 


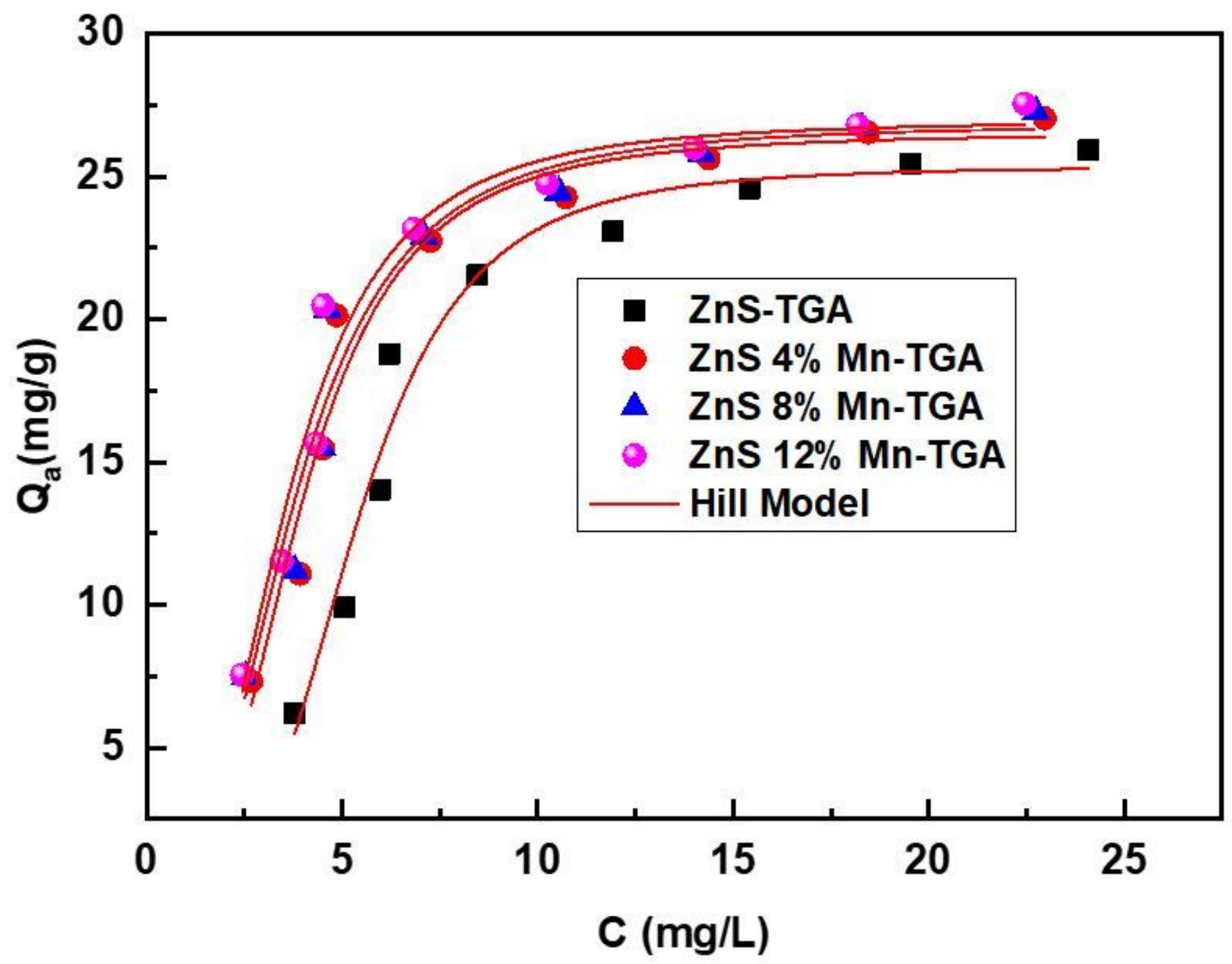

Figure 12

Isotherms of MO adsorption on Mn-doped ZnS-TGA nanocrystals at room temperature 

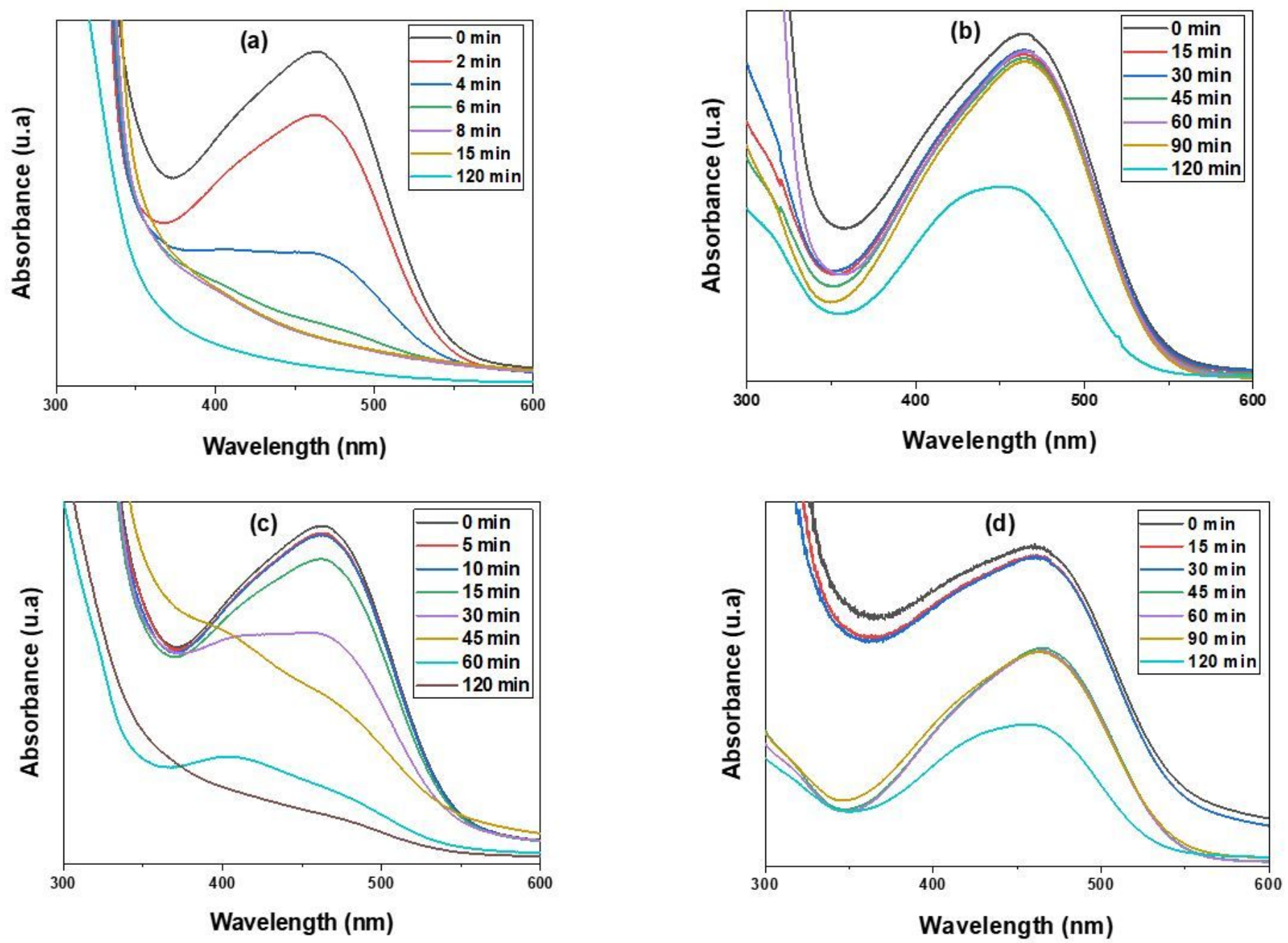

Figure 13

UV-visible absorption spectra of MO solution after photodegradation with (a) undoped and (b-d) Mndoped ZnS-TGA nanocrystals under UV irradiation. 

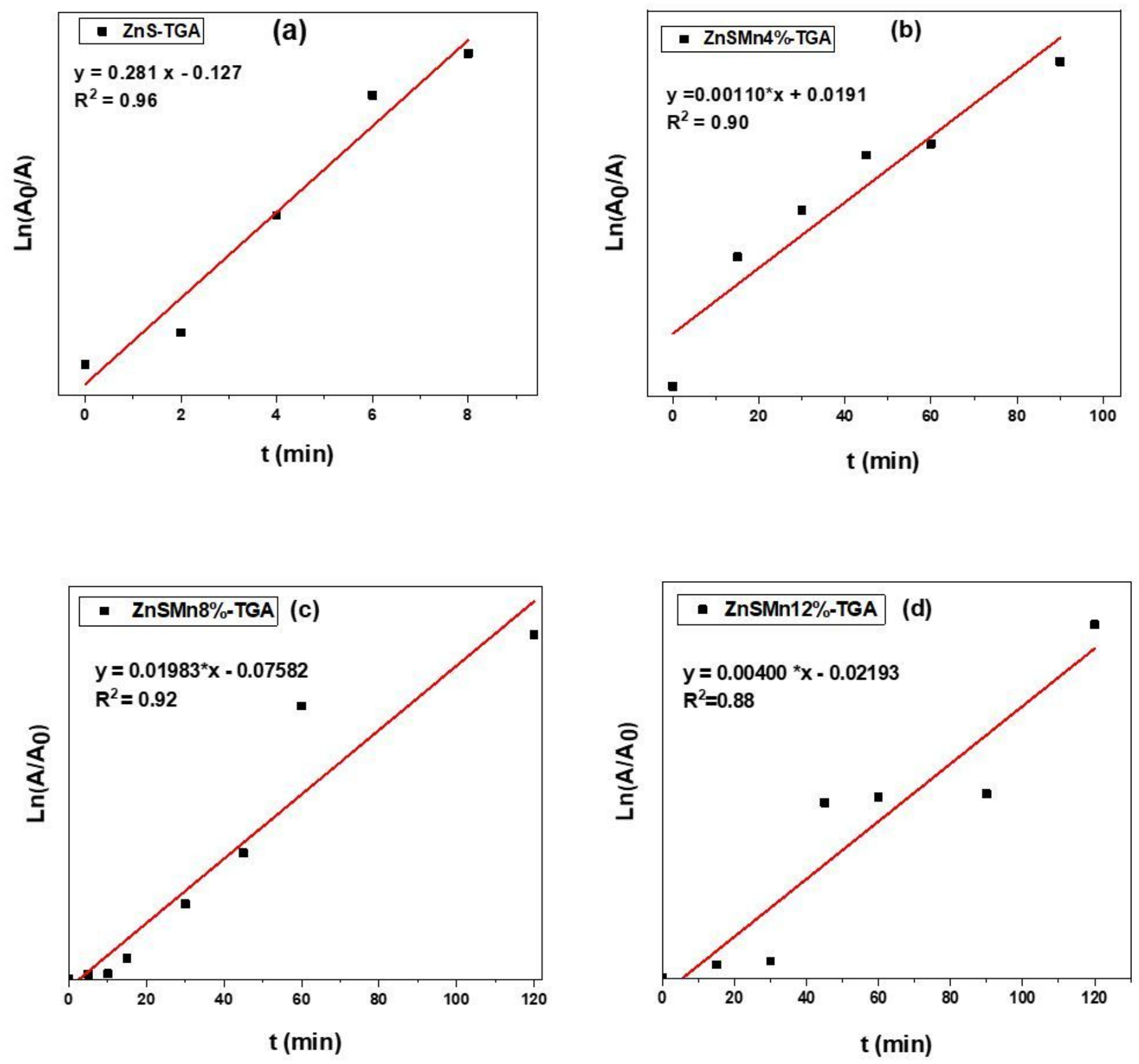

Figure 14

Data correlation for the calculation of degradation rate constants for (a) undoped and (b-d) Mn-doped ZnS-TGA nanocrystals. 

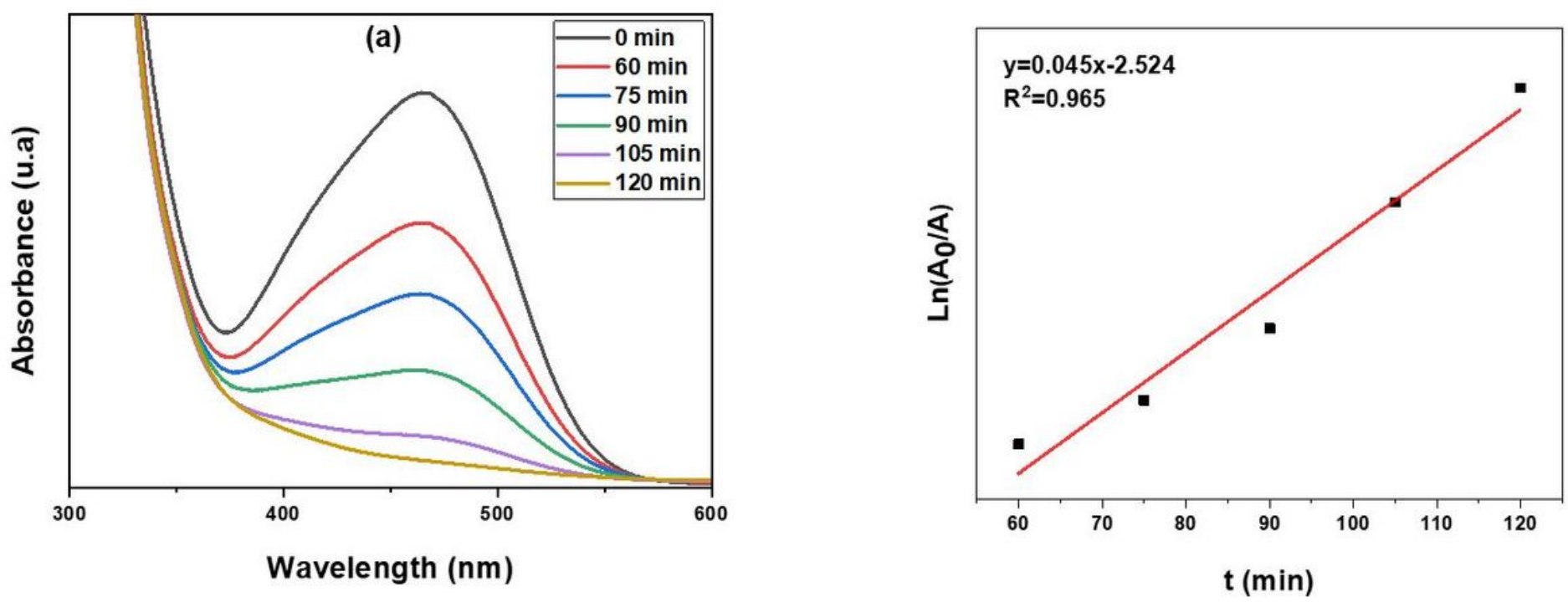

Figure 15

(a) UV-visible absorption spectra of MO solution after photodegradation using ZnS-TGA under sunlight and (b) data correlation for the calculation of degradation rate constant.

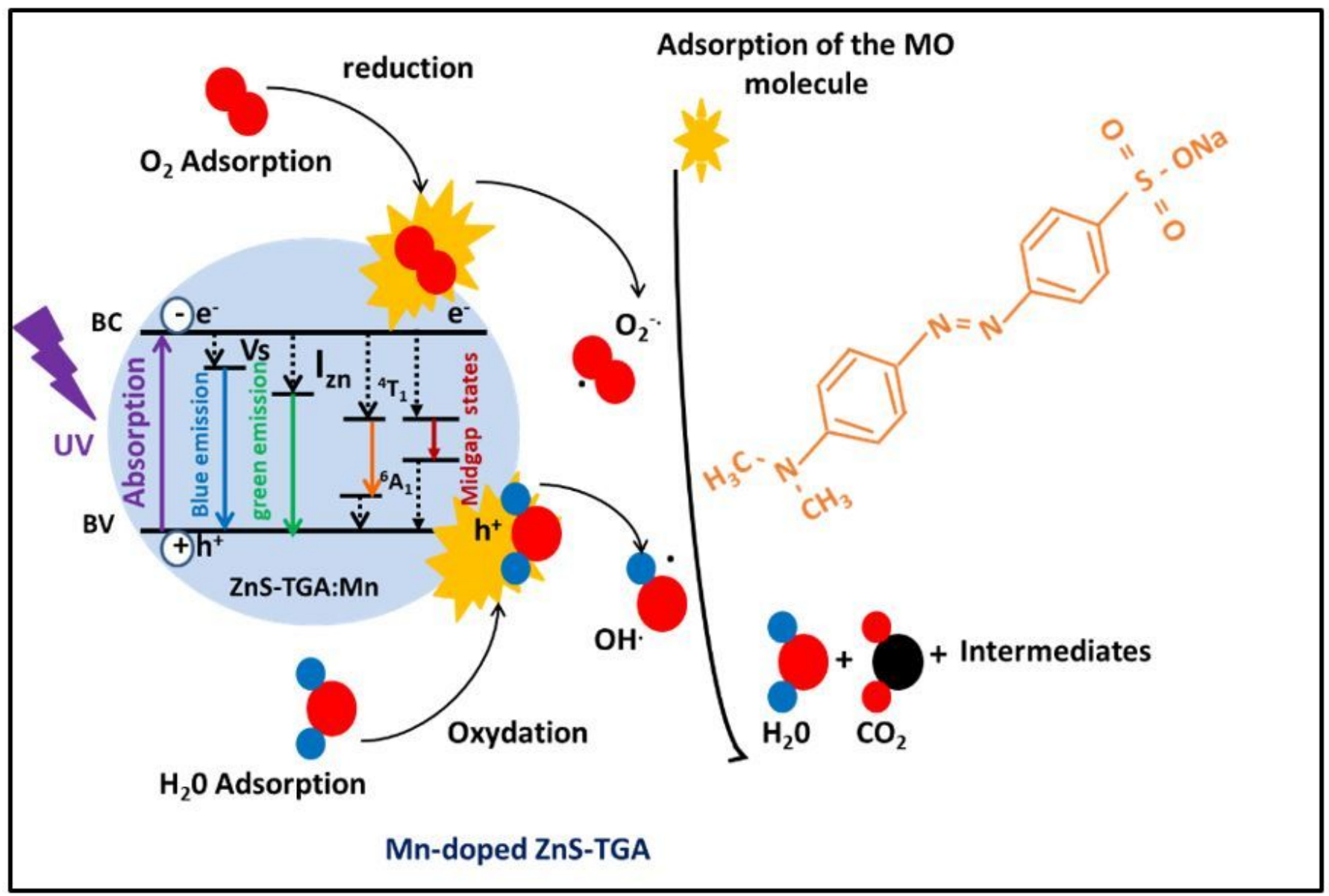

Figure 16 
Illustration of the mechanism of degradation of MO using Mn-doped ZnS-TGA nanocrystals. 